Disponível em:

http://editora.unoesc.edu.br/index.php/race

RACE, Joaçaba, v. 17, n. 2, p. 535-566, maio/ago. 2018

\title{
TEORIA DO PROSPECTO: FATORES DETERMINANTES NAS PREFERÊNCIAS AO RISCO NO BRASIL
}

\author{
Prospect Theory: determinant factors in risk preferences in Brazil
}

Robert Eugene Lobel

E-mail: relobel@gmail.com

Mestre em Ciências da Computação pela Pontifícia Universidade Católica do Rio de Janeiro; Especialista em Finanças pela Pontifícia Universidade Católica do Rio de Janeiro; aposentado.

Marcelo Cabus Klotzle

E-mail: klotzle@iag.puc-rio.br

Pós-doutor em Finanças Comportamentais pela McMaster University; Doutor em Economia pela Katholische Universitat Eichstatt; Professor associado na Pontifícia Universidade Católica do Rio de Janeiro.

Paulo Vitor Jordão da Gama Silva E-mail: rjdagama@hotmail.com

Mestre em Administração (Finanças) pela Pontifícia Universidade Católica do Rio de Janeiro; Doutorando em Finanças pela Pontifícia Universidade Católica do Rio de Janeiro; Professor na Universidade Federal Rural do Rio de Janeiro. Endereço para contato: Rodovia BR 465, Km 07, s/n, Zona Rural, 23890-000, Seropédica, Rio de Janeiro, Brasil.

\section{Antonio Carlos Figueiredo Pinto}

E-mail: figueiredo@iag.puc-rio.br Doutor em Economia pela Escola de Pós-graduação em Economia da Fundação Getúlio Vargas - RJ; Graduado em Administração pela Universidade do Estado do Rio de Janeiro; Professor no Instituto de Administração e Gerência da Pontifícia Universidade Católica do Rio de Janeiro.

Artigo recebido em 21 de outubro de 2017. Aceito em 19 de maio de 2018. 


\section{Resumo}

A preferência ao risco é um fator importante que influencia uma ampla gama de decisões financeiras pessoais (SNELBECKER; ROSZKOWSKI; CUTLER, 1990) e é definida como a quantidade máxima de incerteza que alguém está disposto a aceitar ao tomar uma decisão financeira ou a disposição de se envolver em comportamentos cujos resultados são incertos com possibilidade de se ter um resultado negativo identificável (IRWIN, 1993). Nesse contexto, a Teoria do Prospecto surge como um modelo alternativo descritivo de escolha sob incerteza. Tendo em vista a importância crescente da influência de aspectos comportamentais no ambiente financeiro, neste estudo buscou-se analisar as preferências ao risco no Brasil seguindo os preceitos da Teoria do Prospecto. Para tal, por meio de questionários de loterias (utilizadas no estudo de RIEGER; WANG; HENS, 2011), foram estimados para uma amostra de estudantes e profissionais, os parâmetros das funções valor (com inclusão da função logarítmica modificada) e peso, supondo diversas formas funcionais, para então associar esses parâmetros a determinadas variáveis sociodemográficas. Não foram encontrados estudos precedentes com o objetivo de realizar essa análise no Brasil, sendo que com base na literatura revisada, pôde-se perceber similaridades e divergências. A análise por gênero, estado civil, faixa etária e renda mostra relativas similaridades com outros estudos em países desenvolvidos e em desenvolvimento. Entretanto, o nível educacional mostra resultados contrários ao esperado e a análise por profissão chega a resultados inconclusivos.

Palavras-chave: Finanças comportamentais. Teoria do Prospecto. Função valor. Função peso. Brasil.

\section{Abstract}

Risk preference is an important factor that influence a wide range of personal financial decisions (SNELBECKER; ROSZKOWSKI; CUTLER, 1990) and is defined as the maximum amount of uncertainty that someone is willing to accept when making a financial decision or the disposition of to be involved in behaviors whose results are uncertain with the possibility of having an identifiable negative result (IRWIN, 1993). In this context, the Prospect Theory emerges like a descriptive alternative model of choice under uncertainty. Given the increasing importance of the influence of behavioral aspects in the financial environment, this study sought to analyze the risk preferences in Brazil following the precepts of the Prospect Theory. To this end, with lottery questionnaires (used in the study by RIEGER; WANG; HENS, 2011), were estimated for a sample of students and professionals, parameters of value (with inclusion of the modified logarithmic function) and weight functions assuming various functional forms, and then associate these parameters to certain socio-demographic variables. No previous studies were found in order to perform this analysis in Brazil, and based on the literature reviewed, we could realize some similarities and differences. Analysis by gender, marital status, age and income shows similarities with other studies in developed and developing countries. However, 
the educational level shows results contrary to expected and the analysis by profession comes to inconclusive results.

Keywords: Behavioral finance. Prospect Theory. Value function. Weight function. Brazil.

\section{INTRODUÇÃO}

Durante muito tempo modelos tradicionais de finanças foram baseados na economia neoclássica. Esta, por sua vez, parte de algumas suposições sobre o comportamento do tomador de decisão, entre elas: preferências racionais, maximização da utilidade esperada e posse de todas as informações disponíveis.

Por outro lado, estudos recentes em finanças comportamentais (BARBERIS, 2013; BARBERIS;THALER, 2003; DE BONDT, 1998) mostraram evidências que a Teoria do Prospecto (KAHNEMAN; TVERSKY, 1979) e a Teoria do Prospecto Cumulativa (TVERSKY; KAHNEMAN, 1992) fornecem uma melhor descrição das escolhas do investidor do que o modelo de média/variância de Markowitz (1952).

Grande parte dos estudos utilizou amostras de estudantes para testar a tomada de decisões sob a ótica da Teoria do Prospecto (ABDELLAOUI; BLEICHRODT; L'HARIDON, 2008; HARRISON; RUTSTRÖM, 2009; ZEISBERGER; VRECKO; LANGER, 2012). Outros estudos (GUREVICH; KLIGER; LEVY, 2009; KLIGER; LEVY, 2009; ABDELLAOUI; BLEICHRODT; KAMMOUN, 2011; DICHTL; DROBETZ, 2011) tentaram analisar se profissionais do mercado financeiro atuam de acordo com as premissas da Teoria da Utilidade Esperada ou agem de acordo com a Teoria do Prospecto. Nesses trabalhos foram estimados os parâmetros das funções valor e peso com base em técnicas paramétricas e/ou não paramétricas, em muitos casos, associados com variáveis sociodemográficas.

Grande parte desses estudos (com essa metodologia e tipologia de aplicação) foi feita com base em amostras de países desenvolvidos e, em menor escala, em países em desenvolvimento. Além de existir um déficit de estudos modelando curvas de valor e peso em países em desenvolvimento e em especial no Brasil, faltam estudos que associem tais parâmetros a variáveis sociodemográficas. Isso é de extrema importância quando se pretende entender quais fatores estão por trás das tomadas de decisão.

Com base nisso, nesta pesquisa procura-se contribuir para o estudo de finanças comportamentais no Brasil, ao auxiliar na análise das preferências ao risco com base no gênero, idade, classe econômica, estado civil, nível profissional e nível de educação, no ambiente brasileiro. 


\section{REFERENCIAL TEÓRICO}

\subsection{TEORIA DO PROSPECTO}

A Teoria do Prospecto elaborada por Tversky e Kahneman (KAHNEMAN; TVERSKY, 1979) apresenta uma alternativa à Teoria da Utilidade Esperada, ao introduzir uma função de distorção da probabilidade observada ("função de peso") e uma função de valor que expressa a variação da riqueza.

Ao longo dos últimos 30 anos, variantes a essa Teoria vêm sendo propostas, como a Teoria do Prospecto Cumulativa, desenvolvidas pelos próprios Tversky e Kahneman (TVERSKY; KAHNEMAN, 1992) e a Teoria do Prospecto Normalizada (RIEGER; WANG, 2008; KARMARKAR, 1979; KARMARKAR, 1978). Essas alternativas englobam variações na modelagem teórica, na função de peso e na função de valor.

Kahneman e Tversky (1979) propuseram, com a Teoria do Prospecto, um modelo alternativo descritivo de escolha sob incerteza. Conforme exemplificado em Camerer (2000), a Teoria do Prospecto pode prever corretamente escolhas individuais, até mesmo nos casos em que a Teoria da Utilidade Esperada é violada (ALLAIS, 1953; ELLSBERG, 1961).

Na Teoria do Prospecto, a função valor $(v(x))$ substitui a função utilidade na Teoria da Utilidade Esperada. De acordo com Kahneman e Tversky (1979), a função valor $(v)$ pode ser parametrizada como uma função potência, da seguinte forma:

$$
v(\mathrm{x})= \begin{cases}x^{\alpha}, & x \geq 0 \\ -\lambda(-x)^{\beta}, & x<0\end{cases}
$$

Em que $\alpha$ e $\beta$ medem a curvatura da função valor para ganhos e perdas respectivamente, e $\lambda$ é o coeficiente de aversão à perda.

Uma segunda característica da Teoria do Prospecto se refere à estimativa de probabilidades sobre a ocorrência de eventos. Enquanto a Teoria da Utilidade Esperada usa probabilidades simples, a Teoria do Prospecto usa pesos de decisão. Tversky e Kahneman (1992) definiram e calibraram com base em experimentos uma função peso, que associa a cada probabilidade $p$ um peso $w(p)$. Esse peso reflete, por sua vez, o impacto de $p$ no valor total do prospecto. Na maioria dos casos a soma dos pesos é menor que 1 , ou seja, $w(p)+w(p-1)<1$. 
A função peso $(w(p))$ é parametrizada da seguinte forma:

$$
w(p)=\frac{p^{\gamma}}{\left(p^{\gamma}+(1-p) \gamma\right)^{\frac{1}{\gamma}}},
$$

Em que $\gamma \varepsilon(0,1)$. Uma característica dessa função peso é que ela dá um peso maior a baixas e um peso menor a altas probabilidades. O valor de $\gamma$ irá determinar o grau de sobre ou subavaliação do peso dado às probabilidades absolutas. Quanto menor esse parâmetro, maior será a distorção das probabilidades, já que grande parte da função ficará abaixo da linha de $45^{\circ}$.

De acordo com a Teoria do Prospecto, o valor de um prospecto para uma loteria com $x_{i}$ resultados, cada um com probabilidade $p_{i}$, pode ser definido como (RIEGER; BUI, 2011):

$$
v(x, p)=w\left(p_{1}\right) v\left(x_{1}\right)+w\left(p_{2}\right) v\left(x_{2}\right)+\cdots+w\left(p_{n}\right) v\left(x_{n}\right),
$$

Em que $w(p)$ é a função peso e $v(x)$ é a função valor.

Em 1992, Tversky e Kahneman (1992) apresentaram uma nova versão da Teoria do Prospecto, que chamaram de Teoria do Prospecto Cumulativa. A principal diferença em relação à Teoria do Prospecto foi a inclusão das distorções de probabilidade nas probabilidades acumuladas em vez das individuais, com a intenção de incluir preferências não lineares (rankdependence) e satisfazer a condição de dominância estocástica.

Dessa maneira, pode-se definir o valor de um prospecto para uma loteria com $x_{i}$ resultados, cada um com probabilidade $p_{i}$, como:

$$
v(x, p)=\sum_{i=1}^{n} w\left(p_{i}\right) v\left(x_{i}\right)
$$

Em que $v(x)$ é a função valor, como na Teoria do Prospecto, e $w(p)$ é a função peso subjetiva derivada das probabilidades dos resultados, definida como (RIEGER; BUI, 2011):

$$
w\left(p_{i}\right)=w\left(p_{1}+\cdots+p_{n}\right)-w\left(p_{1}+\cdots+p_{i-1}\right) \text { para } 1<i<n,
$$

Uma variação da Teoria do Prospecto é conhecida como Teoria do Prospecto Normalizada e foi aprimorada por Rieger e Wang (2008), baseada em Karmarkar 
(1978). O valor de um prospecto para uma loteria com $x_{i}$ resultados, cada um com probabilidade $p_{i}$, é definido como:

$$
v(x, p)=\frac{\sum_{i=1}^{n} w\left(p_{i}\right) v\left(x_{i}\right)}{\sum_{i=1}^{n} w\left(p_{i}\right)},
$$

Nesse caso, a função prospecto é normalizada pela soma das probabilidades subjetivas. Essa normalização permite estender a Teria do Prospecto para loterias não discretas.

Ao longo do tempo, diversos tipos de funções foram sugeridos dentro da formulação teórico-empírica da Teoria do Prospecto. Estes envolvem diferentes especificações da função valor e da função peso.

Referente à função valor, vale destacar que além da função potência usada por Kahneman e Tversky (1979) e definida em (1), as funções logarítmicas e quadráticas são também citadas na literatura (RIEGER; BUI, 2011).

A função logarítmica é definida como (KÖBBERLING; WAKKER, 2005):

$$
v(x)=\left\{\begin{array}{l}
\frac{\ln (1+\alpha x)}{(1.0001-\alpha)}, x \geq 0 \\
-\frac{\ln (1+\beta x)}{(1.0001-\beta)}, x<0
\end{array},\right.
$$

A função quadrática, por sua vez, é definida da seguinte maneira:

$$
v(\mathrm{x})=\left\{\begin{array}{c}
x-\alpha x^{2}, x \geq 0 \\
\lambda\left(x+\beta x^{2}\right), x<0
\end{array}\right.
$$

A função exponencial, por sua vez, é definida da seguinte maneira:

$$
v(\mathrm{x})=\left\{\begin{array}{l}
1-e^{-\alpha x}, \quad x \geq 0 \\
-\lambda\left(1-e^{\beta x}\right), x<0
\end{array},\right.
$$

A função peso apresenta também algumas variações funcionais além da desenvolvida por Tversky e Kahneman (1992) e definida em (2). Podem-se citar, entre estas, as funções propostas por Karmarkar (1978), Karmarkar (1979) e Prelec (1998). 
A função peso de Karmarkar (KARMARKAR, 1978; KARMARKAR, 1979) é definida como:

$$
w(p)=\frac{p^{\gamma}}{\left(p^{\gamma}+(1-p)^{\gamma}\right)^{\prime}}
$$

Prelec (1998), por sua vez, propôs a forma composta invariante da função peso, caracterizada como:

$$
w(p)=\exp \left(-(-\ln (p))^{\gamma}\right.
$$

Funções de probabilidade com dois parâmetros também foram desenvolvidas. Entre as mais importantes podem ser citadas as funções de Goldstein e Einhorn (1987) e de Prelec (1998).

A função peso de dois parâmetros desenvolvida por Goldstein e Einhorn (1987) é definida como:

$$
\pi(p)=\frac{\delta p^{\gamma}}{\left(\delta p^{\gamma}+(1-p)^{\gamma}\right)^{\prime}}
$$

Em que $\gamma$ é o coeficiente que controla a curvatura e $\delta$ é o coeficiente que controla a elevação da função.

A função desenvolvida por Prelec (1998), por sua vez, é definida como:

$$
\pi(p)=\exp \left(-\delta(-\ln (p))^{\gamma}\right.
$$

Em que $\gamma$ e $\delta$ são definidos como em (12).

\subsection{TEORIA DO PROSPECTO E VARIÁVEIS DEMOGRÁFICAS}

Fehr-Duda, Gennaro e Schubert (2006) exploraram a relação entre o gênero e a função peso, concluindo que as mulheres são mais avessas ao risco que os homens, além de exibirem um maior pessimismo na área de ganhos. Além do mais, mulheres exibem uma maior distorção da probabilidade do que os homens, o que implica um menor y para as mulheres, resultado também comprovado por Charupat et al. (2013). 
Resultados semelhantes em relação ao risco foram encontrados por Borghans et al. (2009), Croson e Gneezy (2009), Booij, Van Praag e Van De Kuilen (2010) e Von Gaudecker, Van Soest e Wengstrom (2011), que chegaram à conclusão que mulheres são mais avessas ao risco do que homens. Von Gaudecker, Van Soest e Wengstrom (2011) comprovaram, além disso, que mulheres exibem um maior coeficiente de aversão à perda $(\lambda)$ do que o gênero masculino.

Booij, Van Praag e Van De Kuilen (2010) analisaram o efeito da idade sobre os parâmetros da função valor e peso e chegaram a resultados interessantes. Pessoas mais velhas parecem avaliar o dinheiro mais linearmente, pois uma diferença de 50 anos está associada em média a uma potência que é 0.15 maior. Esse efeito, apesar de trabalhar para reduzir a aversão ao risco, é contrabalanceado por um menor parâmetro de distorção das probabilidades ( $\gamma$ cai em 0.30), o que, por sua vez, aumenta a aversão ao risco. O efeito total desses estimadores depende, no entanto, do tipo de prospecto. Para prospectos que possuem uma pequena probabilidade de se obter um ganho bem alto, a aversão ao risco cai com a idade, ao passo que em jogos que não possuem essa característica, uma maior aversão ao risco está associada a uma maior idade.

A relação entre idade e aversão ao risco é controversa na literatura. Estudos como os de Pålsson (1996), Donkers e Van Soest (1999), Halek e Eisenhauer (2001), Hartog, Ferrer-I-Carbonell e Jonker (2002) e Von Gaudecker, Van Soest e Wengstrom (2011) encontraram uma relação positiva entre idade e risco. Outros estudos como os de Barsky et al. (1997), Cohen e Einav (2007), Harrison, Lau e Rutström (2007) e Noussair, Trautmann e Van De Kuilen (2014) encontraram, por sua vez, uma relação não linear (forma de U) entre idade e aversão ao risco. De acordo com esses estudos a aversão ao risco diminui até certa idade, quando ela inverte e volta a aumentar.

No estudo de Cohen e Einav (2007), por exemplo, a aversão ao risco diminui entre 18 e 48 anos à medida que as pessoas envelhecem, quando a partir de 48 anos a situação se inverte, ou seja, à medida que as pessoas vão envelhecendo aumenta a aversão ao risco. Resultado semelhante é encontrado no estudo de Harrison, Lau e Rutström (2007), que analisaram atitudes frente ao risco na Dinamarca. Nesse estudo o grupo de pessoas na meia idade (entre 40 e 50 anos) exibia uma menor aversão ao risco que aqueles com pessoas mais novas e mais velhas.

Estudos como os de Harrison, Lau e Rutström (2007) e Von Gaudecker, Van Soest e Wengstrom (2011) encontraram uma relação negativa entre nível educacional e aversão ao risco. Resultados semelhantes são encontrados por Donkers, Melenberg e Van Soest (2001), Hartog, Ferrer-I-Carbonell e Jonker (2002) e Dohmen et al. (2005), que demonstram que a aversão ao risco diminui com os anos de estudo. Uma explicação 
para isso pode vir de uma maior sensibilidade à perda, à medida que os anos de estudo aumentam, conforme documentado em Gächter, Johnson e Herrmann (2010). Isso é comprovado por Booij, Van Praag e Van De Kuilen (2010), que demonstraram que a formação educacional está associada a um menor coeficiente de aversão à perda $(\lambda)$.

Alguns estudos analisam também a renda e sua relação com a aversão ao risco. Tais estudos encontram uma relação negativa entre aumento da renda e aversão ao risco, como é o caso dos estudos de Donkers, Melenberg e Van Soest (2001), Hartog, Ferrer-I-Carbonell e Jonker (2002) e Noussair, Trautmann e Van De Kuilen (2014). Outros estudos como o de Booij, Van Praag e Van De Kuilen (2010) e de Gächter, Johnson e Herrmann (2010) ainda encontram uma relação negativa entre renda e aversão à perda $(\lambda)$. Existem outras variáveis que foram analisadas em alguns estudos, que serão abordadas aqui com vistas a dar mais consistência a este trabalho. Estas incluem profissão e estado civil.

Em relação à profissão existem poucos estudos que analisam sua relação com a tomada de risco sob incerteza. Entretanto, vale a pena citar o estudo de Abdellaoui, Bleichrodt e L'Haridon (2013) que analisaram para um grupo de profissionais do mercado financeiro se estes se comportavam de acordo com a Teoria do Prospecto. Os autores obtiveram um suporte claro para a Teoria do Prospecto, demonstrando que tais profissionais se comportavam de acordo com a Teoria do Prospecto e violavam a premissa da maximização da utilidade esperada. Eles eram avessos ao risco na área de ganhos e propensos ao risco na área de perdas, e sua utilidade era côncava para ganhos e levemente convexa para perdas. Eles eram também avessos à perda, mas menos do que comumente observado em estudos de laboratório.

Uma comparação com outro estudo feito com estudantes por Abdellaoui, Bleichrodt e Paraschiv (2007) e que usou a mesma metodologia de estimação, mostrou que apesar de ambos os grupos exibirem um coeficiente de inclinação parecido na área de ganhos ( $\alpha_{\text {estudantes }}=0,72 \approx \alpha_{\text {profissionais }}=0,73$ ), a inclinação na área de perdas era menor para os estudantes do que para os profissionais do mercado financeiro $\left(\beta_{\text {estudantes }}=0,73<\beta_{\text {profissionais }}=0,86\right)$. A menor inclinação na área de perdas implica que os profissionais do mercado financeiro têm uma curva de utilidade menos convexa nesse domínio, contribuindo para uma menor propensão ao risco quando confrontados somente com perdas. Por fim, os profissionais do mercado financeiro exibiam um menor coeficiente de aversão à perda $(\lambda)$ do que os estudantes, contribuindo para uma maior propensão ao risco em jogos mistos, ou seja, que envolviam tanto ganhos quanto perdas. 
O estado civil, por sua vez, foi abordado em alguns estudos envolvendo sua relação com o risco. Ao contrário do esperado, Grable (2000) chegou à conclusão que pessoas casadas exibem uma maior tolerância ao risco financeiro que pessoas solteiras. Por outro lado, Sundén e Surette (1998) constataram que homens e mulheres casados são mais avessos ao risco que pessoas do mesmo gênero solteiras.

As evidências apontam que o casamento induz a um aumento da aversão ao risco. Dados sobre comportamento familiar indicam, por exemplo, que uma mudança do estado civil de solteiro para casado está associada a uma queda do percentual investido em ações e outros ativos de risco, indicando um aumento da aversão ao risco (LOVE, 2010).

Roussanov e Savor (2013), por sua vez, analisaram o impacto do casamento em diretores executivos de empresas e concluíram que firmas gerenciadas por executivos solteiros exibiam uma maior volatilidade de suas ações no mercado de capitais, possuíam uma política de investimentos mais agressiva e não respondiam a mudanças no risco idiossincrático. Esse impacto diminuía, entretanto, com o aumento da idade dos gerentes.

\section{METODOLOGIA}

\subsection{AMOSTRA E QUESTIONÁRIO}

O trabalho de pesquisa foi realizado por meio da plataforma on-line Qualtrics, que reuniu inicialmente um grupo de 251 respondentes por meio de pesquisas em universidades brasileiras, empresas e redes sociais. Após realizados os filtros de inconsistência, que serão descritos a seguir, restaram 75 respondentes efetivos para a participação nos processos de análise.

Aos participantes do estudo foram apresentadas as loterias utilizadas no estudo de Rieger, Wang e Hens (2011), que são replicadas na Tabela 1: 
Tabela 1 - Prospectos utilizados no estudo

\begin{tabular}{rrrrrr}
\hline Loteria & $\begin{array}{r}\text { Resultado } \\
\text { A (\$) }\end{array}$ & Prob (A) & $\begin{array}{r}\text { Resultado } \\
\text { B (\$) }\end{array}$ & Prob (B) & $\begin{array}{r}\text { Valor médio } \\
\text { (\$) }\end{array}$ \\
\hline 1 & 10 & 0,1 & 100 & 0,9 & 91 \\
2 & 0 & 0,4 & 100 & 0,6 & 60 \\
3 & 0 & 0,1 & 100 & 0,9 & 90 \\
4 & 0 & 0,4 & 10.000 & 0,6 & 6.000 \\
5 & 0 & 0,9 & 100 & 0,1 & 10 \\
6 & 0 & 0,4 & 400 & 0,6 & 240 \\
7 & -80 & 0,6 & 0 & 0,4 & -48 \\
8 & -100 & 0,6 & 0 & 0,4 & -60 \\
9 & -25 & 0,5 & - & 0,5 & - \\
10 & -100 & 0,5 & - & 0,5 & - \\
\hline
\end{tabular}

Nas primeiras 6 loterias são calculadas as preferências ao risco na área de ganhos. Para tal foi perguntada aos participantes sua propensão a pagar por essas loterias. As loterias têm resultados binários em R\$, junto com a probabilidade de ocorrer cada resultado. As loterias foram estruturadas combinando diferentes níveis de resultados ( $\mathrm{R} \$ 10, \mathrm{R} \$ 100, \mathrm{R} \$ 400, \mathrm{R} \$ 10.000)$ com diferentes níveis de probabilidade $(0,1,0,4,0,5,0,6$ e 0,9$)$.

Segue a forma como foram feitas as perguntas na área de ganhos, tendo como exemplo a loteria 1 :

Jogo 1: a) 10\% de chance de ganhar $\mathrm{R} \$ 10,00$. b) $90 \%$ de ganhar $\mathrm{R} \$ 100,00$.

Qual o valor máximo que você pagaria para participar deste jogo?

Com o intuito de distinguir a área de propensão ao risco da área de aversão ao risco, a segunda medida advém de duas loterias (7 e 8) examinando a atitude perante ao risco na área de perdas. Aos participantes foi instruído que eles tinham que imaginar serem obrigados a jogar na loteria, a não ser que pagassem um valor em dinheiro antecipadamente. Esse montante de dinheiro é o valor negativo da propensão a pagar, também conhecido como propensão a aceitar.

Segue a forma como foram feitas as duas perguntas na área de perdas, tendo como base a loteria 7:

Jogo 7: a) 40\% de chance de ganhar R\$ 0,00. ) 60\% de perder R\$ 80,00. 
Qual o valor máximo que você pagaria para não participar deste jogo?

A terceira medida, após os sujeitos apreçarem as 8 loterias, serve para o cálculo do coeficiente de aversão à perda. Ela é baseada nas loterias 9 e 10 (loterias mistas) e pergunta o valor mínimo $\mathrm{R} \$ X$ de ganho que o participante aceitaria receber, caso aceitasse uma aposta em que ele teria 50\% de chance de perder um valor determinado.

A seguir é demonstrada a forma como foram feitas as duas perguntas na área de jogos mistos, tendo como base a loteria 9:

Jogo 9: a) 50\% de chance de perder $\mathrm{R} \$ 25,00$

b) $50 \%$ de ganhar uma determinada quantia.

Qual o valor mínimo que você exige para querer participar do jogo?

Por sua vez, para filtrar a base de dados dos jogos e torná-la mais robusta, foram adotadas as seguintes regras de consistência para excluir loterias individuais da amostra: se no Jogo 1 o valor de resposta for menor ou igual a $\mathrm{R} \$ 10,00$ ou se for maior ou igual a R\$ 100,00; se o valor obtido no Jogo 3 for maior que o valor obtido no Jogo 1; se os Jogos 2 ou 5 forem maiores que R\$100,00; se o valor no Jogo 7 for igual ou superior a R\$ 80,00; se o valor no Jogo 8 for igual ou superior a R\$ 100,00; se o valor obtido no Jogo 7 for maior que o valor obtido no Jogo 8; se o valor obtido no Jogo 9 for maior que $\mathrm{R} \$ 500,00$ e se o valor obtido no Jogo 10 for superior a $\mathrm{R} \$$ 2.000,00; se o valor obtido no Jogo 9 for inferior a $\mathrm{R} \$ 5,00$ e se o valor obtido no Jogo 10 for inferior a R\$ 20,00; se o valor obtido no Jogo 2 for igual a R\$100,00, se o valor obtido no Jogo 5 for igual a R\$100,00 e se o valor obtido no Jogo 6 for de R\$400,00.

Em relação à replicação das loterias usadas no estudo de Rieger, Wang e Hens (2011), alguns estudos sugerem que seja feita a conversão em moeda local usando o Poder de Paridade de Compra de cada país (HARRISON; HUMPHREY; VERSCHOOR, 2010; RIEGER; BUI, 2011). Entretanto, vale a pena citar que nesses estudos foi feita uma comparação entre países, o que não é o caso deste estudo. Como exemplo pode ser citado o estudo de Tanaka, Camerer e Nguyen (2010) que, analisando preferências ao risco no Vietnã, aplicaram o jogo direto na moeda local, tendo como base a renda média da amostra. Se for tomado como base o rendimento nominal domiciliar per capita médio do brasileiro de R\$ 1.052,00 em 2014 (IBGE, 2014), os valores monetários das loterias parecem bem realistas para o objetivo proposto. 
Com a intenção de incluir um número maior de participantes, optou-se por não fazer este estudo em laboratório, mas por meio de questionário. Em razão disso não foi dado nenhum incentivo monetário para os participantes. Apesar de a discussão sobre a efetividade de incentivos monetários não fazer parte do escopo deste trabalho, vale a pena salientar que alguns autores, incluindo Tversky e Kahneman (1992) e Camerer (1989), não encontraram nenhuma diferença significativa com a inclusão dos incentivos. Isso está de acordo também com o estudo de Etchart-Vincent e L’Haridon (2011), que demonstraram que, pelo menos na área de perdas, a inclusão do incentivo financeiro não exerce nenhum impacto nas escolhas dos indivíduos.

\subsection{ESTIMAÇÃO DOS PARÂMETROS E MODELOS DE REGRESSÃO}

Neste estudo as estimações das funções valor e peso serão feitas de forma paramétrica, pois se baseará em funções valor e peso definidas a priori.

Serão consideradas como base para a estimação as funções valor na forma potência e exponencial, definidas em (1) e (9). A função quadrática definida em (8) não será abordada neste estudo. No seu lugar será proposta uma nova função, denominada logarítmica modificada, na forma a seguir:

$$
v(\mathrm{x})=\left\{\begin{array}{c}
\frac{\ln (1+\alpha x)}{\alpha}, x \geq 0 \\
-\lambda\left(\frac{\ln (1+\beta x)}{\beta}\right), x<0
\end{array},\right.
$$

No caso da função peso serão consideradas as funções Tversky-Kahneman, Karmarkar e Prelec, definidas em (2), (10) e (11).

Este estudo partirá, portanto, de três teorias definidas respectivamente em (3), (5) e (6) (Teoria do Prospecto - PT; Teoria do Prospecto Cumulativa - CPT; Teoria do Prospecto Normalizada (NPT), três funções valor (potência, exponencial e logarítmica modificada) e três funções peso (Tversky-Kahneman - TK; Karmarkar e Prelec) e o modelo abordado na teoria da utilidade, gerando 28 modelos, conforme a Tabela 2: 
Robert Eugene Lobel et al.

Tabela 2 - Modelos utilizados

\begin{tabular}{|c|c|c|c|}
\hline Modelo & Teoria & $w(p)$ & $v(x)$ \\
\hline \multicolumn{4}{|l|}{ TUE } \\
\hline 111 & PT & TK & Potência \\
\hline 112 & & & Exponencial \\
\hline 113 & & & Log Modificada \\
\hline 121 & & Karmarkar & Potência \\
\hline 122 & & & Exponencial \\
\hline 123 & & & Log Modificada \\
\hline 131 & & Prelec & Potência \\
\hline 132 & & & Exponencial \\
\hline 133 & & & Log Modificada \\
\hline 211 & $\mathrm{CPT}$ & TK & Potência \\
\hline 212 & & & Exponencial \\
\hline 213 & & & Log Modificada \\
\hline 221 & & Karmarkar & Potência \\
\hline 222 & & & Exponencial \\
\hline 223 & & & Log Modificada \\
\hline 231 & & Prelec & Potência \\
\hline 232 & & & Exponencial \\
\hline 233 & & & Log Modificada \\
\hline 311 & NPT & TK & Potência \\
\hline 312 & & & Exponencial \\
\hline 313 & & & Log Modificada \\
\hline 321 & & Karmarkar & Potência \\
\hline 322 & & & Exponencial \\
\hline 323 & & & Log Modificada \\
\hline 331 & & Prelec & Potência \\
\hline 332 & & & Exponencial \\
\hline 333 & & & Log Modificada \\
\hline
\end{tabular}

Fonte: adaptada de Rieger, Wang e Hens (2011).

Nota: Para simplificação foram adotadas as seguintes abreviações ao longo do texto, além das usadas na Tabela: Karmarkar: KAR; Prelec: PRC; Potência: PWR; Exponencial: EXP; Log Modificada: LOG.

Em linha com o estudo de Bui (2009), neste estudo utiliza-se a metodologia grid search para estimar todos os parâmetros das funções peso e valor, com o intuito de minimizar a soma dos erros. Os parâmetros serão estimados para cada indivíduo, sendo que a função erro é definida como a soma das diferenças entre o equivalente certo e as respostas de todas as 10 loterias, conforme a Equação 15: 


$$
\text { Erros }=\sum_{L=1}^{10} \frac{\left|C E_{i}-x_{i}\right|}{\max \left(\left|A_{i}\right|\left|B_{i}\right|\right)}, L=\{1,2, \ldots 10\},
$$

Em que o valor $\mathrm{x}_{\mathrm{i}}$ é definido como a resposta de cada indivíduo aos dados, que é a propensão a pagar se $x_{i} \geq 0$, e a propensão a aceitar (valor negativo da propensão a pagar) se $x_{i}<0$. Matematicamente, o objetivo pode ser definido como:

$$
\text { Ótimo }(\alpha, \beta, \gamma)=\min (\alpha, \beta, \gamma) \sum_{L=1}^{10} \frac{\left|C E_{i}-x_{i}\right|}{\max \left(\left|A_{i}\right|\left|B_{i}\right|\right)}, L=\{1,2, \ldots 10\} \text {, }
$$

Por sua vez, o Equivalente Certo ( $\left.\mathrm{CE}_{i}\right)$ é definido para cada loteria como o inverso da função valor no resultado do cálculo do prospecto (Yi) para PT, CPT e NPT:

$$
C E_{i}=v^{-1}\left(Y_{i}\right)
$$

O valor de $\lambda$, por sua vez, é estimado usando-se os prospectos 9 e 10, calculando a relação entre a função valor na região de ganhos e a função valor na região de perdas. Repare-se que como o CE dessas loterias é definido como 0 , o valor $\lambda$ é calculado com base nos valores $\alpha, \beta$ e $\gamma$ das respostas $X_{9}$ e $X_{10}$ e dos valores dos prospectos $\mathrm{A}_{9}$ e $\mathrm{A}_{10}$, com probabilidade 0,5.

A modelagem econométrica consiste em estimar modelos de regressão simples regredindo os parâmetros $\alpha, \beta$, $\gamma$ e $\lambda$ respectivamente contra cada variável demográfica. Estas foram modeladas como variáveis dummy (categóricas).

A modelagem das equações individuais contendo as variáveis dummy será feita sem a inclusão de intercepto, conforme a Equação 18:

$$
Y_{i}=y_{1} D_{1}+y_{2} D_{2}+\cdots+y_{n} D_{n}+\epsilon_{i},
$$

Em que $Y_{t}$ é a variável dependente, nesse caso, os parâmetros $\alpha, \beta$, $\gamma$ e $\lambda, D_{1} \ldots$ $D_{n}$ são as categorias das respectivas variáveis demográficas e é o resíduo da regressão. Como não será incluído o intercepto, os coeficientes das variáveis dummy refletirão o valor esperado de cada parâmetro em relação à categoria analisada. Vale ressaltar que a regressão múltipla não foi considerada em razão do tamanho pequeno da amostra. 


\section{RESULTADOS}

Primeiramente em relação às regressões realizadas, vale apontar que foram testados os pressupostos para a robustez das regressões. O teste de White comprovou homoscedasticidade, o teste de Ljung-Box não detectou autocorrelação e o teste de Jarque-bera confirmou a normalidade dos resíduos.

Analisando a Tabela 3, que se refere ao gênero, pode-se perceber que na maioria dos modelos, na área de ganhos as mulheres são mais avessas ao risco do que os homens (menor $\alpha$ ). Na área de perdas, por sua vez, não se pode constatar uma predominância da aversão ao risco (menor $\beta$ ) das mulheres. Já a distorção das probabilidades é claramente maior nas mulheres (maior $\gamma$ ), mostrando um maior realismo que os homens. Por fim, segundo o $\lambda$, nota-se que as mulheres são claramente mais avessas à perda do que os homens, o que vai de encontro à revisão de literatura tanto em países desenvolvidos quanto em desenvolvimento.

O que mais chama a atenção é o maior realismo nas mulheres, que contrasta com estudos como o de Charupat et al. (2013), e a menor aversão ao risco das mulheres na área de perdas. Estudos como o de Fehr-Duda, Gennaro e Schubert (2006), que usaram a função peso de dois fatores de Prelec (1998) encontraram um maior pessimismo das mulheres que os homens na área de ganhos. Uma explicação possível para a menor aversão ao risco das mulheres na área de perdas no Brasil poderia, portanto, estar associada a um maior otimismo delas. No estudo de Braga (2015), inclusive, o autor avaliou comportamento de investidores no mercado de ações brasileiro e notou que as mulheres declararam assumir perdas muito menores do que homens, exigem ganhar tanto quanto eles e ganham bem menos do que gostariam.

Tabela 3 - Análise levando em consideração o gênero

\begin{tabular}{rrrrrrrrr}
\hline \multirow{3}{*}{ Modelo } & \multicolumn{4}{c}{ Mulheres } & \multicolumn{4}{c}{ Homens } \\
\cline { 2 - 9 } & $\boldsymbol{\alpha}$ & $\boldsymbol{\beta}$ & $\boldsymbol{\gamma}$ & $\boldsymbol{\lambda}$ & $\mathbf{A}$ & $\mathbf{B}$ & $\gamma$ & $\boldsymbol{\lambda}$ \\
\hline 111 & $0,54^{*}$ & $0,62^{*}$ & $0,59^{*}$ & $2,13^{*}$ & $0,52^{*}$ & $0,79^{*}$ & $0,48^{*}$ & $0,79^{*}$ \\
112 & $0,07^{* *}$ & $0,23^{*}$ & $0,83^{*}$ & $1,50^{*}$ & $0,10^{*}$ & $0,05^{* *}$ & $0,71^{*}$ & $1,63^{*}$ \\
113 & $0,21^{*}$ & $0,36^{*}$ & $0,61^{*}$ & $2,51^{*}$ & $0,31^{*}$ & $0,07^{*}$ & $0,49^{*}$ & $1,19^{*}$ \\
121 & $0,46^{*}$ & $0,46^{*}$ & $0,37^{*}$ & $2,13^{*}$ & $0,34^{*}$ & $0,47^{*}$ & $0,22^{*}$ & $0,97^{*}$ \\
122 & $0,08^{*}$ & $0,24^{*}$ & $0,85^{*}$ & $1,37^{*}$ & $0,11^{*}$ & $0,07^{*}$ & $0,68^{*}$ & $1,38^{*}$ \\
123 & $0,35^{*}$ & $0,47^{*}$ & $0,40^{*}$ & $2,54^{*}$ & $0,71^{*}$ & $0,35^{*}$ & $0,24^{*}$ & $1,03^{*}$ \\
131 & $0,53^{*}$ & $0,56^{*}$ & $0,38^{*}$ & $2,33^{*}$ & $0,41^{*}$ & $0,60^{*}$ & $0,24^{*}$ & $0,89^{*}$ \\
132 & $0,07^{* *}$ & $0,23^{*}$ & $0,81^{*}$ & $1,47^{*}$ & $0,12^{*}$ & $0,07^{*}$ & $0,76^{*}$ & $1,39^{*}$ \\
133 & $0,22^{* *}$ & $0,34^{*}$ & $0,43^{*}$ & $2,54^{*}$ & $0,47^{*}$ & $0,18^{*}$ & $0,27^{*}$ & $1,06^{*}$ \\
\hline
\end{tabular}




\begin{tabular}{|c|c|c|c|c|c|c|c|c|}
\hline \multirow{2}{*}{ Modelo } & \multicolumn{4}{|c|}{ Mulheres } & \multicolumn{4}{|c|}{ Homens } \\
\hline & $\alpha$ & $\beta$ & $Y$ & $\lambda$ & A & B & $Y$ & $\lambda$ \\
\hline 211 & $0,33^{*}$ & $0,36^{*}$ & $0,66^{*}$ & $1,11^{*}$ & $0,21 *$ & $0,32 *$ & $0,57^{*}$ & $0,54^{*}$ \\
\hline 212 & 0,15 & $0,25 *$ & $0,87^{*}$ & $1,02^{*}$ & $0,27 *$ & $0,08^{*}$ & $0,81^{*}$ & $1,11^{*}$ \\
\hline 213 & $0,73^{*}$ & $0,64^{*}$ & $0,77^{*}$ & $1,25^{*}$ & $0,85^{*}$ & $0,61^{*}$ & $0,72 *$ & $0,96^{*}$ \\
\hline 221 & $0,46^{*}$ & $0,46 *$ & $0,37 *$ & $2,13^{*}$ & $0,34^{*}$ & $0,47^{*}$ & $0,22 *$ & $0,97^{*}$ \\
\hline 222 & $0,08^{*}$ & $0,24 *$ & $0,85^{*}$ & $1,37^{*}$ & $0,11^{*}$ & $0,07^{*}$ & $0,68^{*}$ & $1,38^{*}$ \\
\hline 223 & $0,35 *$ & $0,47^{*}$ & $0,40 *$ & $2,54 *$ & $0,71 *$ & $0,35 *$ & $0,24 *$ & $1,03^{*}$ \\
\hline 231 & $0,36^{*}$ & $0,38 *$ & $0,50 *$ & $1,24 *$ & $0,26 *$ & $0,37 *$ & $0,29 *$ & $0,67^{*}$ \\
\hline 232 & $0,09 *$ & $0,24 *$ & $0,94 *$ & $1,32 *$ & $0,15^{*}$ & $0,08 *$ & $0,91 *$ & $1,27^{*}$ \\
\hline 233 & $0,66^{*}$ & $0,65^{*}$ & $0,55^{*}$ & $1,24^{*}$ & $0,84^{*}$ & $0,64^{*}$ & $0,37^{*}$ & $0,87^{*}$ \\
\hline 311 & $0,46^{*}$ & $0,46 *$ & $0,38 *$ & $2,13^{*}$ & $0,34^{*}$ & $0,47^{*}$ & $0,23 *$ & $0,97^{*}$ \\
\hline 312 & $0,08^{*}$ & $0,24^{*}$ & $0,85^{*}$ & $1,37^{*}$ & $0,11^{*}$ & $0,07^{*}$ & $0,68^{*}$ & $1,38^{*}$ \\
\hline 313 & $0,35 *$ & $0,48^{*}$ & $0,37 *$ & $2,55^{*}$ & $0,71^{*}$ & $0,35^{*}$ & $0,21^{*}$ & $1,03^{*}$ \\
\hline 321 & $0,46^{*}$ & $0,46^{*}$ & $0,37 *$ & $2,13^{*}$ & $0,34^{*}$ & $0,47^{*}$ & $0,22 *$ & $0,97^{*}$ \\
\hline 322 & $0,08^{*}$ & $0,24^{*}$ & $0,85^{*}$ & $1,37^{*}$ & $0,11^{*}$ & $0,07 *$ & $0,68^{*}$ & $1,38^{*}$ \\
\hline 323 & $0,35 *$ & $0,47^{*}$ & $0,40^{*}$ & $2,54^{*}$ & $0,71^{*}$ & $0,35^{*}$ & $0,24 *$ & $1,03^{*}$ \\
\hline 331 & $0,45^{*}$ & $0,46^{*}$ & $0,37^{*}$ & $2,09 *$ & $0,34^{*}$ & $0,47^{*}$ & $0,21^{*}$ & $0,97^{*}$ \\
\hline 332 & $0,07 *$ & $0,24^{*}$ & $0,82 *$ & $1,38^{*}$ & $0,11^{*}$ & $0,07 *$ & $0,62 *$ & $1,38^{*}$ \\
\hline \multirow[t]{2}{*}{333} & $0,35 *$ & $0,48^{*}$ & $0,37 *$ & $2,55^{*}$ & $0,71^{*}$ & $0,35^{*}$ & $0,21 *$ & $1,03^{*}$ \\
\hline & \multicolumn{8}{|c|}{ * 1\%; ** 5\%; *** 10\%; variáveis em negrito não são significantes. } \\
\hline
\end{tabular}

Fonte: os autores.

Em relação à regressão pelo estado civil (Tabela 4), pode-se perceber que na maioria dos modelos pessoas casadas são mais avessas ao risco tanto na área de ganhos quanto na área de perdas do que pessoas solteiras e divorciadas, além de exibirem uma maior aversão à perda. Analisando o parâmetro $\gamma$, pode-se deduzir, por sua vez, que as pessoas divorciadas são mais realistas ao perceberem a probabilidade. Estudos discutidos anteriormente apoiam os resultados encontrados. O interessante é notar pouca diferença na distorção de probabilidades entre homens solteiros e casados.

Tabela 4 - Análise levando em consideração o estado civil

\begin{tabular}{|c|c|c|c|c|c|c|c|c|c|c|c|c|}
\hline \multirow{2}{*}{$\begin{array}{l}\text { Mo- } \\
\text { delo }\end{array}$} & \multicolumn{4}{|c|}{ Solteiro(a) } & \multicolumn{4}{|c|}{ Divorciado(a) } & \multicolumn{4}{|c|}{ Casado(a) } \\
\hline & $\alpha$ & B & $\Gamma$ & $\lambda$ & A & $\beta$ & $Y$ & $\Lambda$ & $\alpha$ & $\boldsymbol{\beta}$ & $Y$ & $\Lambda$ \\
\hline 111 & $0,54^{*}$ & $0,77^{*}$ & $0,49 *$ & $1,01^{*}$ & $0,43 *$ & $0,72 *$ & $0,57 *$ & $1,10 *$ & $0,52 *$ & $0,77^{*}$ & $0,48^{*}$ & $0,97 *$ \\
\hline 112 & $0,09 *$ & $0,07 * *$ & $0,73 *$ & $1,77^{*}$ & $0,17^{*}$ & 0,05 & $0,83^{*}$ & $1,22 *$ & $0,09 *$ & $0,11^{*}$ & $0,71^{*}$ & $1,45^{*}$ \\
\hline 113 & $0,29 *$ & $0,10^{*}$ & $0,51 *$ & $1,32 *$ & $0,45^{*}$ & 0,12 & $0,54^{*}$ & $1,09 * *$ & $0,26^{*}$ & $0,15^{*}$ & $0,50 *$ & $1,62 *$ \\
\hline 121 & $0,37 *$ & $0,47^{*}$ & $0,25 *$ & $1,18^{*}$ & $0,37 *$ & $0,52 *$ & $0,22 * * *$ & $1,29 *$ & $0,34^{*}$ & $0,46^{*}$ & $0,25^{*}$ & $1,08^{*}$ \\
\hline 122 & $0,09 *$ & $0,09 *$ & $0,66 *$ & $1,44^{*}$ & $0,15 *$ & 0,05 & $0,76^{*}$ & $1,26^{*}$ & $0,11^{*}$ & $0,13^{*}$ & $0,76^{*}$ & $1,31^{*}$ \\
\hline
\end{tabular}




\begin{tabular}{|c|c|c|c|c|c|c|c|c|c|c|c|c|}
\hline \multirow{2}{*}{$\begin{array}{l}\text { Mo- } \\
\text { delo }\end{array}$} & \multicolumn{4}{|c|}{ Solteiro(a) } & \multicolumn{4}{|c|}{ Divorciado(a) } & \multicolumn{4}{|c|}{ Casado(a) } \\
\hline & $\alpha$ & B & $\Gamma$ & $\lambda$ & $\mathbf{A}$ & $\beta$ & $Y$ & $\Lambda$ & $\alpha$ & $\beta$ & $Y$ & $\Lambda$ \\
\hline 123 & $0,65^{*}$ & $0,36^{*}$ & $0,26^{*}$ & $1,19 *$ & $0,74 *$ & $0,35 *$ & $0,26 * *$ & $1,26 * *$ & $0,63^{*}$ & $0,40^{*}$ & $0,26 *$ & $1,42^{*}$ \\
\hline 131 & $0,44 *$ & $0,59 *$ & $0,27^{*}$ & $1,14^{*}$ & $0,41 *$ & $0,65 *$ & $0,27 * *$ & $1,17^{*}$ & $0,42^{*}$ & $0,58 *$ & $0,25 *$ & $1,08^{*}$ \\
\hline 132 & $0,11^{*}$ & $0,08 *$ & $0,76^{*}$ & $1,47^{*}$ & $0,16 *$ & 0,05 & $0,78 *$ & $1,23^{*}$ & $0,11^{*}$ & $0,12^{*}$ & $0,76^{*}$ & $1,32^{*}$ \\
\hline 133 & $0,38^{*}$ & $0,19 *$ & $0,30 *$ & $1,27 *$ & $0,64 *$ & 0,16 & $0,29 * *$ & $1,10^{* *}$ & $0,44^{*}$ & $0,23^{*}$ & $0,29 *$ & $1,40^{*}$ \\
\hline 211 & $0,23 *$ & $0,34 *$ & $0,59 *$ & $0,57 *$ & $0,25^{* *}$ & $0,35 *$ & $0,58 *$ & $0,81^{*}$ & $0,21^{*}$ & 0,30 * & $0,58 *$ & $0,70^{*}$ \\
\hline 212 & $0,21^{*}$ & $0,10^{*}$ & $0,85^{*}$ & $1,16^{*}$ & $0,39 *$ & 0,07 & $0,73 *$ & $0,80 *$ & $0,29 *$ & $0,14^{*}$ & $0,80 *$ & $1,07^{*}$ \\
\hline 213 & $0,82 *$ & $0,63^{*}$ & $0,73^{*}$ & $0,93 *$ & $0,76 *$ & $0,60 *$ & $0,75^{*}$ & $1,28 *$ & $0,87^{*}$ & $0,59 *$ & $0,72 *$ & $1,06^{*}$ \\
\hline 221 & $0,37 *$ & $0,47^{*}$ & $0,25^{*}$ & $1,18^{*}$ & $0,37 *$ & $0,52 *$ & $0,22 * * *$ & $1,29 *$ & $0,34 *$ & $0,46^{*}$ & $0,25 *$ & $1,08^{*}$ \\
\hline 222 & $0,09 *$ & $0,09 *$ & $0,66^{*}$ & $1,44^{*}$ & $0,15^{*}$ & 0,05 & $0,76^{*}$ & $1,26^{*}$ & $0,11^{*}$ & $0,13^{*}$ & $0,76^{*}$ & $1,31^{*}$ \\
\hline 223 & $0,65^{*}$ & $0,36^{*}$ & $0,26^{*}$ & $1,19 *$ & $0,74 *$ & $0,35^{*}$ & $0,26 * *$ & $1,26^{* *}$ & $0,63^{*}$ & $0,40^{*}$ & $0,26^{*}$ & $1,42^{*}$ \\
\hline 231 & $0,29 *$ & $0,38 *$ & $0,33^{*}$ & $0,72 *$ & $0,29 *$ & $0,40 *$ & $0,31^{* *}$ & $0,88^{*}$ & $0,26^{*}$ & $0,35^{*}$ & $0,32 *$ & $0,80^{*}$ \\
\hline 232 & $0,13^{*}$ & $0,10 *$ & $0,92 *$ & $1,36^{*}$ & $0,19 *$ & 0,06 & $0,88^{*}$ & $1,10^{*}$ & $0,13^{*}$ & $0,14^{*}$ & $0,91 *$ & $1,18^{*}$ \\
\hline 233 & $0,81^{*}$ & $0,66 *$ & $0,39 *$ & $0,86^{*}$ & $0,77^{*}$ & $0,61^{*}$ & $0,44 *$ & $1,10^{*}$ & $0,82 *$ & $0,62^{*}$ & $0,40^{*}$ & $1,02^{*}$ \\
\hline 311 & $0,37 *$ & $0,47^{*}$ & $0,25^{*}$ & $1,18^{*}$ & $0,36 *$ & $0,52 *$ & $0,22 * * *$ & $1,29 *$ & $0,34 *$ & $0,46^{*}$ & $0,25 *$ & $1,09^{*}$ \\
\hline 312 & $0,09 *$ & $0,09 *$ & $0,66^{*}$ & $1,44^{*}$ & $0,15 *$ & 0,05 & $0,76^{*}$ & $1,26^{*}$ & $0,11^{*}$ & $0,13^{*}$ & $0,76^{*}$ & $1,31^{*}$ \\
\hline 313 & $0,65^{*}$ & $0,36 *$ & $0,26^{*}$ & $1,19 *$ & $0,74 *$ & $0,35 *$ & $0,27 * *$ & $1,26^{* *}$ & $0,63^{*}$ & $0,40^{*}$ & $0,26 *$ & $1,42^{*}$ \\
\hline 321 & $0,37 *$ & $0,47 *$ & $0,25^{*}$ & $1,18^{*}$ & $0,37 *$ & $0,52 *$ & $0,22 * * *$ & $1,29 *$ & $0,34^{*}$ & $0,46^{*}$ & $0,25 *$ & $1,08^{*}$ \\
\hline 322 & $0,65^{*}$ & $0,36 *$ & $0,24 *$ & $1,19 *$ & $0,74 *$ & $0,35 *$ & $0,26 * *$ & $1,26^{* *}$ & $0,64^{*}$ & $0,40^{*}$ & $0,24 *$ & $1,43^{*}$ \\
\hline 323 & $0,65^{*}$ & $0,36 *$ & $0,26^{*}$ & $1,19 *$ & $0,74 *$ & $0,35 *$ & $0,26 * *$ & $1,26^{* *}$ & $0,63^{*}$ & $0,40^{*}$ & $0,26 *$ & $1,42^{*}$ \\
\hline 331 & $0,36 *$ & $0,47^{*}$ & $0,23^{*}$ & $1,17^{*}$ & $0,36 *$ & $0,51^{*}$ & $0,25 * * *$ & $1,29 *$ & $0,34^{*}$ & $0,46^{*}$ & $0,23 *$ & $1,07^{*}$ \\
\hline 332 & $0,09 *$ & $0,09 *$ & $0,62 *$ & $1,44^{*}$ & $0,14 *$ & 0,05 & $0,69 *$ & $1,26^{*}$ & $0,10^{*}$ & $0,13^{*}$ & $0,70 *$ & $1,31^{*}$ \\
\hline \multirow[t]{2}{*}{333} & $0,65^{*}$ & $0,36 *$ & $0,24 *$ & $1,19 *$ & $0,74 *$ & $0,35 *$ & $0,26 * *$ & $1,26 * *$ & $0,64 *$ & $0,40^{*}$ & $0,24 *$ & $1,43^{*}$ \\
\hline & & & & & & \multicolumn{7}{|c|}{ * 1\%; ** 5\%; *** $10 \%$; variáveis em negrito não são significantes } \\
\hline
\end{tabular}

Fonte: os autores.

Já a análise pela faixa etária (Tabela 5) mostra uma tendência de pessoas mais velhas serem mais conservadoras, o que vai parcialmente ao encontrado na literatura. Pode-se perceber que na maioria dos modelos na área de ganhos $(\alpha)$ as pessoas na faixa dos 32 aos 38 anos são mais avessas ao risco do que as demais, seguidas pelas pessoas na faixa dos 46 ou >. Já na área de perdas ( $\beta$ ) as pessoas na faixa dos 46 ou > apresentam maior aversão ao risco do que as pessoas mais novas. Por sua vez, segundo o parâmetro $\gamma$, pode-se notar que as pessoas na faixa dos 46 ou > são mais realistas ao perceberem a probabilidade, e, finalmente segundo o $\lambda$, nota-se que as pessoas na faixa dos 39 aos 45 são mais avessas à perda, seguidas das pessoas na faixa dos 46 ou >.

Analisando o nível educacional (Tabela 6), pode-se deduzir que um maior nível educacional está associado a uma maior aversão ao risco na área de ganhos, e em parte, na área de perdas. Isso também pode ser constatado em relação ao parâmetro $\gamma$, 
sendo que pessoas com maior nível educacional apresentam uma menor distorção das probabilidades. O resultado mais interessante é pessoas com nível de doutorado apresentarem a maior aversão à perda do que os outros grupos. Esses resultados contestam a maioria dos estudos realizados até agora e discutidos anteriormente.

Já a análise segundo as classes sociais distribuídas pela renda (Tabela 7) mostra que, em geral, pessoas com o menor nível de riqueza são mais avessos ao risco, distorcem mais as probabilidades e são mais avessas à perda que as demais, o que vai de encontro aos estudos realizados tanto em países desenvolvidos quanto em desenvolvimento.

Por fim, a análise pela profissão é inconclusiva. Analisando a Tabela 8, pode-se perceber que, segundo o $\alpha$, em média pessoas em cargos de gerência são mais avessas ao risco do que as demais; segundo o $\beta$, em média pessoas em cargos de assistente possuem maior aversão ao risco na área da perda; segundo o $\gamma$, pode-se notar que pessoas em cargos de gerência são mais realistas; e em $\lambda$, nota-se que pessoas em cargos de supervisão ou coordenação são mais avessas à perda do que as demais, nessa categoria. 
Tabela 5 - Análise levando em conta a idade

\begin{tabular}{|c|c|c|c|c|c|c|c|c|c|c|c|c|c|c|c|c|c|c|c|c|}
\hline \multirow{2}{*}{$\begin{array}{l}\text { Mo- } \\
\text { delo }\end{array}$} & \multicolumn{4}{|c|}{ Entre 18 e 24 anos } & \multicolumn{4}{|c|}{ Entre 25 e 31 anos } & \multicolumn{4}{|c|}{ Entre 32 e 38 anos } & \multicolumn{4}{|c|}{ Entre 39 e 45 anos } & \multicolumn{4}{|c|}{ Com 46 ou > } \\
\hline & $\alpha$ & $\beta$ & $Y$ & $\lambda$ & $\mathbf{A}$ & $\boldsymbol{\beta}$ & $\gamma$ & $\lambda$ & $\alpha$ & $\beta$ & $Y$ & $\lambda$ & $\alpha$ & $\boldsymbol{\beta}$ & $\Gamma$ & $\lambda$ & $\alpha$ & $\beta$ & $Y$ & $\lambda$ \\
\hline 111 & $0,49 *$ & $0,75^{*}$ & $0,38 *$ & $0,93 *$ & $0,53^{*}$ & $0,79 *$ & $0,52 *$ & $0,91^{*}$ & $0,58 *$ & $0,76^{*}$ & $0,57^{*}$ & $1,14^{*}$ & $0,49 *$ & $0,78^{*}$ & $0,56^{*}$ & $1,25^{* *}$ & $0,51^{*}$ & $0,65^{*}$ & $0,66^{*}$ & $1,24^{* *}$ \\
\hline 112 & $0,14^{*}$ & $0,07 * * *$ & $0,68 *$ & $1,60 *$ & $0,09 *$ & $0,07 * * *$ & $0,75^{*}$ & $1,79 *$ & 0,04 & $0,09 * * *$ & $0,72 *$ & $1,46^{*}$ & $0,12^{*}$ & 0,05 & $0,80 *$ & $1,77^{*}$ & $0,07 * * *$ & $0,22 *$ & $0,86^{*}$ & $0,99 *$ \\
\hline 113 & $0,36^{*}$ & $0,11 * *$ & $0,39 *$ & $1,36^{*}$ & $0,31 *$ & 0,08 & $0,53^{*}$ & $1,16^{*}$ & $0,17 * *$ & $0,14^{* *}$ & $0,58 *$ & $1,51^{*}$ & $0,39 *$ & 0,10 & $0,57 *$ & $1,54 * *$ & $0,23 * * *$ & $0,34 *$ & $0,69 *$ & $2,35 * *$ \\
\hline 121 & $0,27 *$ & $0,38 *$ & 0,06 & $1,05^{*}$ & $0,38 *$ & $0,52 *$ & $0,30 *$ & $1,01^{*}$ & $0,43^{*}$ & $0,53 *$ & $0,32 *$ & $1,47^{*}$ & $0,39 *$ & $0,52 *$ & $0,35^{* *}$ & $1,40^{*}$ & $0,40 *$ & $0,44^{*}$ & $0,57^{*}$ & $1,28 *$ \\
\hline 122 & $0,13 *$ & $0,09 * *$ & $0,56^{*}$ & $1,19 *$ & $0,11^{*}$ & $0,09 * *$ & $0,74 *$ & $1,63^{*}$ & $0,06 *$ & $0,10^{* *}$ & $0,75^{*}$ & $1,28^{*}$ & $0,17 *$ & 0,05 & $0,91^{*}$ & $1,72^{*}$ & $0,08^{* *}$ & $0,23 *$ & $0,86 *$ & $0,97 *$ \\
\hline 123 & $0,86^{*}$ & $0,43 *$ & 0,08 & $0,95^{*}$ & $0,65^{*}$ & $0,37^{*}$ & $0,29 *$ & $1,10^{*}$ & $0,40 *$ & $0,28 *$ & $0,39 *$ & $1,56^{*}$ & $0,64^{*}$ & 0,23 & $0,32 * *$ & $1,66 *$ & $0,47 *$ & $0,52 *$ & $0,51^{*}$ & $2,44 *$ \\
\hline 131 & $0,86^{*}$ & $0,43^{*}$ & 0,08 & $0,95 *$ & $0,65^{*}$ & $0,37 *$ & $0,29 *$ & $1,10^{*}$ & $0,40^{*}$ & $0,28 *$ & $0,39 *$ & $1,56^{*}$ & $0,64^{*}$ & 0,23 & $0,32 * *$ & $1,66^{*}$ & $0,47^{*}$ & $0,52^{*}$ & $0,51^{*}$ & $2,44 *$ \\
\hline 132 & $0,16 *$ & $0,08 * *$ & $0,74^{*}$ & $1,18^{*}$ & $0,11^{*}$ & $0,08^{* *}$ & $0,77^{*}$ & $1,73^{*}$ & $0,06 * *$ & $0,10^{* *}$ & $0,76^{*}$ & $1,20 *$ & $0,14 *$ & 0,04 & $0,76^{*}$ & $1,76^{*}$ & $0,08^{* *}$ & $0,22 *$ & $0,89 *$ & $0,97 *$ \\
\hline 133 & $0,56^{*}$ & $0,23 *$ & $0,13^{* *}$ & $1,07^{*}$ & $0,41^{*}$ & $0,18^{*}$ & $0,32 *$ & $1,11^{*}$ & $0,21^{*}$ & $0,17^{* *}$ & $0,42 *$ & $1,54^{*}$ & $0,57^{*}$ & 0,12 & $0,35^{*}$ & $1,63 * *$ & $0,39 *$ & $0,37 *$ & $0,56^{*}$ & $2,35 * *$ \\
\hline 211 & $0,11^{* *}$ & $0,21^{*}$ & 0,50 * & $0,43^{*}$ & $0,26^{*}$ & $0,38^{*}$ & $0,59 *$ & $0,58 *$ & $0,29 *$ & $0,39 *$ & $0,64^{*}$ & $0,73^{*}$ & $0,29 * *$ & $0,39 *$ & $0,66^{*}$ & $1,21^{*}$ & $0,34 *$ & $0,36^{*}$ & $0,75^{*}$ & $1,02 *$ \\
\hline 212 & $0,31^{*}$ & $0,10^{* *}$ & $0,79 *$ & 0,93* & $0,27^{*}$ & $0,10^{*}$ & $0,80^{*}$ & $1,17^{*}$ & 0,08 & $0,11^{* *}$ & $0,91^{*}$ & $1,24^{*}$ & $0,40 *$ & 0,06 & $0,75^{*}$ & $1,42^{*}$ & $0,23 * * *$ & $0,23 *$ & $0,85^{*}$ & $0,78^{*}$ \\
\hline 213 & $1,00^{*}$ & $0,79 *$ & $0,68^{*}$ & $0,86^{*}$ & $0,78^{*}$ & $0,52^{*}$ & $0,74^{*}$ & $0,92^{*}$ & $0,75^{*}$ & $0,46^{*}$ & $0,73^{*}$ & $0,93^{*}$ & $0,78^{*}$ & $0,62 *$ & $0,76^{*}$ & $1,55^{*}$ & $0,61^{*}$ & $0,66^{*}$ & $0,83^{*}$ & $1,83 *$ \\
\hline 221 & $0,27^{*}$ & $0,38 *$ & 0,06 & $1,05^{*}$ & $0,38^{*}$ & $0,52^{*}$ & $0,30 *$ & $1,01^{*}$ & $0,43^{*}$ & $0,53 *$ & $0,32 *$ & $1,47^{*}$ & $0,39 *$ & $0,52 *$ & $0,35^{* *}$ & $1,40^{*}$ & $0,40 *$ & $0,44^{*}$ & $0,57^{*}$ & $1,28 *$ \\
\hline 222 & $0,13^{*}$ & $0,09 * *$ & $0,56 *$ & $1,19 *$ & $0,11^{*}$ & $0,09 * *$ & $0,74^{*}$ & $1,63^{*}$ & $0,06^{*}$ & $0,10^{* *}$ & $0,75^{*}$ & $1,28^{*}$ & $0,17^{*}$ & 0,05 & $0,91^{*}$ & $1,72^{*}$ & $0,08^{* *}$ & $0,23 *$ & $0,86^{*}$ & $0,97 *$ \\
\hline 223 & $0,86^{*}$ & $0,43^{*}$ & 0,08 & $0,95^{*}$ & $0,65^{*}$ & $0,37 *$ & $0,29 *$ & $1,10^{*}$ & $0,40 *$ & $0,28 *$ & $0,39 *$ & $1,56^{*}$ & $0,64 *$ & 0,23 & $0,32^{* *}$ & $1,66^{*}$ & $0,47^{*}$ & $0,52 *$ & $0,51^{*}$ & $2,44 *$ \\
\hline 231 & $0,18^{*}$ & $0,26^{*}$ & $0,14 * *$ & $0,61 *$ & $0,31^{*}$ & $0,42^{*}$ & $0,34 *$ & $0,71^{*}$ & $0,33^{*}$ & $0,43^{*}$ & $0,46^{*}$ & $0,82^{*}$ & $0,33^{*}$ & $0,42 *$ & $0,39 *$ & $1,25 *$ & $0,36^{*}$ & $0,39 *$ & $0,61^{*}$ & $1,05 *$ \\
\hline 232 & $0,19 *$ & $0,10 * *$ & $0,91^{*}$ & $1,08^{*}$ & $0,13^{*}$ & $0,10 * *$ & $0,90 *$ & $1,55^{*}$ & $0,08^{*}$ & $0,11 * *$ & 0,93* & $1,13^{*}$ & $0,17^{*}$ & 0,05 & $0,91^{*}$ & $1,65^{*}$ & $0,09 * *$ & $0,23^{*}$ & 0,93* & $0,91 *$ \\
\hline 233 & $1,00^{*}$ & $0,83^{*}$ & $0,24 *$ & $0,74 *$ & $0,76^{*}$ & $0,54 *$ & $0,42 *$ & $0,86^{*}$ & $0,69 *$ & $0,48 *$ & $0,50 *$ & $0,87^{*}$ & $0,73^{*}$ & $0,66^{*}$ & $0,48^{*}$ & $1,54^{*}$ & $0,61^{*}$ & $0,67 *$ & $0,65^{*}$ & $1,77 *$ \\
\hline 311 & $0,27 *$ & $0,38 *$ & 0,06 & $1,05^{*}$ & $0,38^{*}$ & $0,52^{*}$ & $0,30 *$ & $1,01^{*}$ & $0,43^{*}$ & $0,53 *$ & $0,32 *$ & $1,47^{*}$ & $0,39 *$ & $0,52 *$ & $0,35^{* *}$ & $1,41^{*}$ & $0,40 *$ & $0,44^{*}$ & $0,57^{*}$ & $1,28 *$ \\
\hline 312 & $0,13^{*}$ & $0,09 * *$ & $0,56^{*}$ & $1,19 *$ & $0,11^{*}$ & $0,09 * *$ & $0,74 *$ & $1,63^{*}$ & $0,06^{*}$ & $0,10^{* *}$ & $0,75^{*}$ & $1,28^{*}$ & $0,17^{*}$ & 0,05 & $0,91^{*}$ & $1,72^{*}$ & $0,08^{* *}$ & $0,23^{*}$ & $0,86^{*}$ & $0,97 *$ \\
\hline 313 & $0,86^{*}$ & $0,43^{*}$ & 0,08 & $0,95 *$ & $0,65^{*}$ & $0,37 *$ & $0,30 *$ & $1,10^{*}$ & $0,40 *$ & $0,28 *$ & $0,40 *$ & $1,56^{*}$ & $0,64 *$ & 0,23 & $0,33 * *$ & $1,66^{*}$ & $0,47^{*}$ & $0,52 *$ & $0,51^{*}$ & $2,44 *$ \\
\hline 321 & $0,27^{*}$ & $0,38 *$ & 0,06 & $1,05^{*}$ & $0,38^{*}$ & $0,52 *$ & $0,30 *$ & $1,01^{*}$ & $0,43^{*}$ & $0,53^{*}$ & $0,32 *$ & $1,47^{*}$ & $0,39 *$ & $0,52^{*}$ & $0,35^{* *}$ & $1,40^{*}$ & $0,40^{*}$ & $0,44^{*}$ & $0,57^{*}$ & $1,28 *$ \\
\hline 322 & $0,13^{*}$ & $0,09 * *$ & $0,56^{*}$ & $1,19 *$ & $0,11^{*}$ & $0,09 * *$ & $0,74^{*}$ & $1,63^{*}$ & $0,06^{*}$ & $0,10^{* *}$ & $0,75^{*}$ & $1,28^{*}$ & $0,17^{*}$ & 0,05 & $0,91^{*}$ & $1,72^{*}$ & $0,08^{* *}$ & $0,23 *$ & $0,86^{*}$ & $0,97 *$ \\
\hline 323 & $0,86^{*}$ & $0,43^{*}$ & 0,08 & $0,95^{*}$ & $0,65^{*}$ & $0,37 *$ & $0,29 *$ & $1,10^{*}$ & $0,40 *$ & $0,28 *$ & $0,39 *$ & $1,56^{*}$ & $0,64 *$ & 0,23 & $0,32^{* *}$ & $1,66^{*}$ & $0,47^{*}$ & $0,52 *$ & $0,51^{*}$ & $2,44 *$ \\
\hline 331 & $0,27 *$ & $0,38 *$ & 0,05 & $1,05^{*}$ & $0,38 *$ & $0,52 *$ & $0,27 *$ & $0,99 *$ & $0,43^{*}$ & $0,53^{*}$ & $0,32 *$ & $1,46^{*}$ & $0,39 *$ & $0,52 *$ & $0,34^{* *}$ & $1,40^{*}$ & $0,40^{*}$ & $0,44^{*}$ & $0,56 *$ & $1,26 *$ \\
\hline 332 & $0,12 *$ & $0,09 * *$ & $0,51^{*}$ & $1,18^{*}$ & $0,10^{*}$ & $0,09 * *$ & $0,69 *$ & $1,64^{*}$ & $0,06^{*}$ & $0,10^{* *}$ & $0,74^{*}$ & $1,27^{*}$ & $0,12 *$ & 0,05 & $0,70^{*}$ & $1,73^{*}$ & $0,08^{*}$ & $0,23 *$ & $0,85^{*}$ & $0,96 *$ \\
\hline 333 & $0,86^{*}$ & $0,43^{*}$ & 0,06 & $0,95^{*}$ & $0,64^{*}$ & $0,37 *$ & $0,27^{*}$ & $1,11^{*}$ & $0,40 *$ & $0,28 *$ & $0,36^{*}$ & $1,58^{*}$ & $0,63^{*}$ & 0,22 & $0,31^{* *}$ & $1,67^{*}$ & $0,47^{*}$ & $0,54^{*}$ & $0,51^{*}$ & $2,43 *$ \\
\hline
\end{tabular}


Tabela 6 - Análise levando em conta a escolaridade

\begin{tabular}{|c|c|c|c|c|c|c|c|c|c|c|c|c|c|c|c|c|c|c|c|c|}
\hline \multirow{2}{*}{$\begin{array}{l}\text { Mo- } \\
\text { delo }\end{array}$} & \multicolumn{4}{|c|}{ Ensino Médio } & \multicolumn{4}{|c|}{ Graduação } & \multicolumn{4}{|c|}{ MBA } & \multicolumn{4}{|c|}{ Mestrado } & \multicolumn{4}{|c|}{ Doutorado } \\
\hline & $\alpha$ & B & $Y$ & $\Lambda$ & $\alpha$ & $\beta$ & $Y$ & $\lambda$ & $\mathbf{A}$ & $\beta$ & $Y$ & $\lambda$ & A & $\boldsymbol{\beta}$ & $Y$ & $\lambda$ & $\alpha$ & $\beta$ & $Y$ & $\lambda$ \\
\hline 111 & $0,68^{*}$ & $0,88^{*}$ & $0,29 * * *$ & 0,83 & $0,50 *$ & $0,73^{*}$ & $0,40 *$ & $0,94 *$ & $0,42 *$ & $0,78^{*}$ & $0,43^{*}$ & $0,86^{*}$ & $0,63^{*}$ & $0,85^{*}$ & $0,77 *$ & $0,79 *$ & $0,62 *$ & $0,66^{*}$ & $0,68^{*}$ & $3,08 *$ \\
\hline 112 & 0,00 & 0,00 & 0,23 & $2,34 * *$ & $0,11^{*}$ & $0,10^{*}$ & $0,68 *$ & $1,52 *$ & $0,14^{*}$ & $0,12 * *$ & $0,76^{*}$ & $1,40 *$ & $0,05^{* *}$ & 0,02 & $0,87 *$ & $1,81^{*}$ & 0,02 & 0,04 & $0,78^{*}$ & $2,12 *$ \\
\hline 113 & 0,32 & 0,07 & $0,39 * *$ & 0,55 & $0,31 *$ & $0,13^{*}$ & $0,42 *$ & $1,43^{*}$ & $0,49 *$ & 0,10 & $0,45^{*}$ & $0,92 * *$ & $0,17 * *$ & 0,04 & $0,76^{*}$ & $1,14^{*}$ & 0,10 & $0,33^{*}$ & $0,69 *$ & $3,82 *$ \\
\hline 121 & 0,26 & $0,33 * * *$ & 0,08 & 0,98 & $0,27 *$ & $0,37 *$ & $0,12 *$ & $1,04 *$ & $0,29 *$ & $0,44 *$ & 0,09 & $1,29 *$ & $0,60 *$ & $0,74^{*}$ & $0,64 *$ & $0,91 *$ & $0,51^{*}$ & $0,52 *$ & $0,53^{*}$ & $2,98 *$ \\
\hline 123 & $0,89 *$ & $0,61^{* * *}$ & 0,02 & 0,94 & $0,81^{*}$ & $0,49 *$ & $0,14^{*}$ & $1,10^{*}$ & $0,76^{*}$ & $0,28 *$ & 0,09 & $1,25^{*}$ & $0,25^{*}$ & 0,09 & $0,63^{*}$ & $1,13^{*}$ & 0,17 & $0,45^{*}$ & $0,63^{*}$ & $3,76^{*}$ \\
\hline 131 & $0,37 * *$ & $0,46^{* *}$ & 0,07 & 0,98 & $0,36 *$ & $0,51^{*}$ & $0,13^{*}$ & $1,00 *$ & $0,37^{*}$ & $0,61^{*}$ & 0,12 & $1,15^{*}$ & $0,63^{*}$ & $0,81^{*}$ & $0,65 *$ & $0,84^{*}$ & $0,59 *$ & $0,59 *$ & $0,53^{*}$ & $3,53 *$ \\
\hline 132 & 0,07 & 0,05 & 0,20 & 1,18 & $0,14^{*}$ & $0,12 *$ & $0,73^{*}$ & $1,18^{*}$ & $0,15^{*}$ & $0,12 * *$ & $0,76^{*}$ & $1,46^{*}$ & $0,05^{* *}$ & 0,02 & $0,85 *$ & $1,82 *$ & 0,04 & 0,05 & $0,92^{*}$ & $1,85^{*}$ \\
\hline 133 & 0,39 & 0,20 & 0,08 & 0,84 & $0,49 *$ & $0,27^{*}$ & $0,18^{*}$ & $1,18^{*}$ & $0,60 *$ & $0,15^{* * *}$ & $0,15^{* *}$ & $1,16^{*}$ & $0,22 *$ & 0,05 & $0,65^{*}$ & $1,14^{*}$ & 0,12 & $0,32 * *$ & $0,59^{*}$ & $3,71^{*}$ \\
\hline 211 & 0,10 & 0,17 & $0,47^{*}$ & 0,40 & $0,12^{*}$ & $0,22 *$ & $0,51^{*}$ & $0,47^{*}$ & $0,13 * *$ & $0,25 *$ & $0,50 *$ & $0,49 *$ & $0,53^{*}$ & $0,66^{*}$ & $0,81^{*}$ & $0,77^{*}$ & $0,43^{*}$ & $0,44^{*}$ & $0,77^{*}$ & $2,30^{*}$ \\
\hline 212 & 0,21 & 0,09 & $0,88^{*}$ & 1,04 & $0,24^{*}$ & $0,14^{*}$ & $0,84^{*}$ & $1,02^{*}$ & $0,53^{*}$ & $0,15^{*}$ & $0,62 *$ & $0,70^{*}$ & $0,14^{* * *}$ & 0,03 & $0,89 *$ & $1,41^{*}$ & 0,04 & 0,06 & $0,95^{*}$ & $1,83^{*}$ \\
\hline 221 & 0,26 & $0,33^{* * *}$ & 0,08 & 0,98 & $0,27^{*}$ & $0,37 *$ & $0,12 *$ & $1,04 *$ & $0,29 *$ & $0,44^{*}$ & 0,09 & $1,29 *$ & $0,60^{*}$ & $0,74^{*}$ & $0,64 *$ & $0,91 *$ & $0,51^{*}$ & $0,52 *$ & $0,53^{*}$ & $2,98^{*}$ \\
\hline 222 & 0,21 & 0,09 & $0,88^{*}$ & 1,04 & $0,24^{*}$ & $0,14^{*}$ & $0,84^{*}$ & $1,02 *$ & $0,53^{*}$ & $0,15^{*}$ & $0,62 *$ & $0,70 *$ & $0,14^{* * *}$ & 0,03 & $0,89 *$ & $1,41^{*}$ & 0,04 & 0,06 & $0,95^{*}$ & $1,83^{*}$ \\
\hline 223 & $0,89 *$ & $0,61^{* * *}$ & 0,02 & 0,94 & $0,81^{*}$ & $0,49 *$ & $0,14^{*}$ & $1,10^{*}$ & $0,76^{*}$ & $0,28^{*}$ & 0,09 & $1,25^{*}$ & $0,25 *$ & 0,09 & $0,63^{*}$ & $1,13^{*}$ & 0,17 & $0,45^{*}$ & $0,63^{*}$ & $3,76^{*}$ \\
\hline 231 & 0,18 & 0,23 & 0,06 & 0,57 & $0,19 *$ & $0,26 *$ & $0,18^{*}$ & $0,64 *$ & $0,19 *$ & $0,31^{*}$ & $0,18^{* *}$ & $0,64 *$ & $0,54^{*}$ & $0,68^{*}$ & $0,71^{*}$ & $0,78^{*}$ & $0,44^{*}$ & $0,45^{*}$ & $0,75^{*}$ & $2,28 *$ \\
\hline 232 & $0,21^{* *}$ & 0,09 & $0,90 *$ & 1,00 & $0,16^{*}$ & $0,13^{*}$ & $0,91^{*}$ & $1,09 *$ & $0,18^{*}$ & $0,13^{* *}$ & $0,87^{*}$ & $1,14^{*}$ & $0,07^{*}$ & 0,02 & $0,96 *$ & $1,77^{*}$ & 0,04 & 0,06 & $0,95^{*}$ & $1,81^{*}$ \\
\hline 233 & $1,00 *$ & $1,00 *$ & 0,23 & 0,82 & $0,96 *$ & $0,81^{*}$ & $0,28 *$ & $0,83^{*}$ & $0,93^{*}$ & $0,56 *$ & $0,30 *$ & $0,67 *$ & $0,38^{*}$ & $0,24 *$ & $0,71^{*}$ & $1,02 *$ & $0,58 *$ & $0,59 *$ & $0,74^{*}$ & $2,40^{*}$ \\
\hline 311 & 0,26 & $0,33^{* * *}$ & 0,08 & 0,98 & $0,27^{*}$ & $0,37 *$ & $0,12 * *$ & $1,05^{*}$ & $0,29 *$ & $0,44^{*}$ & 0,10 & $1,29 *$ & $0,60 *$ & $0,74^{*}$ & $0,64^{*}$ & $0,91 *$ & $0,51^{*}$ & $0,52 *$ & $0,53^{*}$ & $2,98 *$ \\
\hline 312 & 0,09 & 0,07 & 0,18 & 1,09 & $0,12 *$ & $0,12 *$ & $0,60^{*}$ & $1,19 *$ & $0,15^{*}$ & $0,13^{* *}$ & $0,73^{*}$ & $1,26^{*}$ & $0,07^{*}$ & 0,02 & $0,94^{*}$ & $1,87^{*}$ & 0,04 & 0,06 & $0,92 *$ & $1,85^{*}$ \\
\hline 313 & $0,90 *$ & $0,61^{* * *}$ & 0,02 & 0,94 & $0,81^{*}$ & $0,49 *$ & $0,14^{*}$ & $1,10^{*}$ & $0,76^{*}$ & $0,28 *$ & 0,09 & $1,25^{*}$ & $0,25^{*}$ & 0,09 & $0,63^{*}$ & $1,13^{*}$ & 0,17 & $0,45^{*}$ & $0,63^{*}$ & $3,76^{*}$ \\
\hline 321 & 0,26 & $0,33^{* * *}$ & 0,08 & 0,98 & $0,27^{*}$ & $0,37 *$ & $0,12 *$ & $1,04^{*}$ & $0,29 *$ & $0,44^{*}$ & 0,09 & $1,29 *$ & $0,60^{*}$ & $0,74^{*}$ & $0,64 *$ & $0,91 *$ & $0,51^{*}$ & $0,52 *$ & $0,53^{*}$ & $2,98^{*}$ \\
\hline 322 & 0,09 & 0,07 & 0,18 & 1,09 & $0,12^{*}$ & $0,12 *$ & $0,60 *$ & $1,19 *$ & $0,15^{*}$ & $0,13^{* *}$ & $0,73^{*}$ & $1,26^{*}$ & $0,07^{*}$ & 0,02 & $0,94 *$ & $1,87^{*}$ & 0,04 & 0,06 & $0,92^{*}$ & $1,85^{*}$ \\
\hline 323 & $0,89 *$ & $0,61 * * *$ & 0,02 & 0,94 & $0,81^{*}$ & $0,49 *$ & $0,14^{*}$ & $1,10^{*}$ & $0,76^{*}$ & $0,28 *$ & 0,09 & $1,25^{*}$ & $0,25^{*}$ & 0,09 & $0,63^{*}$ & $1,13^{*}$ & 0,17 & $0,45^{*}$ & $0,63^{*}$ & $3,76^{*}$ \\
\hline 332 & $0,10^{* * *}$ & 0,07 & 0,17 & 1,09 & $0,11^{*}$ & $0,13^{*}$ & $0,57 *$ & $1,19 *$ & $0,14^{*}$ & $0,13 * *$ & $0,61^{*}$ & $1,27^{*}$ & $0,05^{*}$ & 0,02 & $0,87^{*}$ & $1,87^{*}$ & 0,04 & 0,06 & $0,92^{*}$ & $1,85^{*}$ \\
\hline 333 & $0,89 *$ & $0,61^{* * *}$ & 0,01 & 0,94 & $0,82 *$ & $0,50 *$ & $0,12 *$ & $1,11^{*}$ & $0,76^{*}$ & $0,28 *$ & 0,07 & $1,25^{*}$ & $0,25^{*}$ & 0,08 & $0,60 *$ & $1,14^{*}$ & 0,17 & $0,47^{*}$ & $0,61^{*}$ & $3,76^{*}$ \\
\hline
\end{tabular}

*1\%; ** 5\%; *** 10\%; variáveis em negrito não são significantes. 


\begin{tabular}{|c|c|c|c|c|c|c|c|c|c|c|c|c|c|c|c|c|c|c|c|c|}
\hline \multirow{2}{*}{$\begin{array}{l}\text { Mo- } \\
\text { delo }\end{array}$} & \multicolumn{4}{|c|}{ Classe A } & \multicolumn{4}{|c|}{ Classe D } & \multicolumn{4}{|c|}{ Classe C } & \multicolumn{4}{|c|}{ Classe B } & \multicolumn{4}{|c|}{ Classe A } \\
\hline & $\alpha$ & $\boldsymbol{\beta}$ & $\gamma$ & $\Lambda$ & $\mathbf{A}$ & $\beta$ & $\gamma$ & $\lambda$ & A & $\boldsymbol{\beta}$ & $Y$ & $\lambda$ & $\alpha$ & $\beta$ & $\gamma$ & $\lambda$ & $\alpha$ & $\beta$ & $Y$ & $\lambda$ \\
\hline 111 & $0,49^{* *}$ & 0,27 & $0,47^{* *}$ & $5,00 *$ & $0,50 *$ & $0,89 *$ & $0,51^{*}$ & 0,54 & $0,48 *$ & $0,72 *$ & $0,47^{*}$ & $1,08^{*}$ & $0,52 *$ & $0,76 *$ & $0,42 *$ & $0,73^{*}$ & $0,57^{*}$ & $0,81^{*}$ & $0,56^{*}$ & $0,97 *$ \\
\hline 112 & 0,06 & $0,41 * *$ & $0,79 *$ & 1,00 & 0,02 & 0,00 & $0,57 *$ & $2,83^{*}$ & $0,11^{*}$ & $0,14^{*}$ & $0,73^{*}$ & $1,48^{*}$ & $0,12 *$ & 0,06 & $0,77^{*}$ & $1,29 *$ & $0,08^{*}$ & 0,03 & $0,73^{*}$ & $1,83^{*}$ \\
\hline 113 & 0,17 & $0,83^{*}$ & $0,47^{* *}$ & $5,00 *$ & 0,29 & 0,04 & $0,56^{*}$ & 0,53 & $0,33^{*}$ & $0,14^{*}$ & $0,47^{*}$ & $1,54^{*}$ & $0,32 *$ & 0,10 & $0,46^{*}$ & $0,89 * *$ & $0,26^{*}$ & $0,09 * *$ & $0,57^{*}$ & $1,44^{*}$ \\
\hline 121 & $0,45^{* *}$ & 0,22 & 0,00 & $5,00 *$ & $0,31^{* * *}$ & $0,58^{*}$ & $0,32 *$ & 0,70 & $0,31^{*}$ & $0,42 *$ & $0,19 *$ & $1,26^{*}$ & $0,32 *$ & $0,42 *$ & 0,14 & $0,90 *$ & $0,42 *$ & $0,55^{*}$ & $0,36^{*}$ & $1,08^{*}$ \\
\hline 122 & 0,07 & $0,45^{* *}$ & $0,90^{*}$ & 1,00 & 0,07 & 0,04 & $0,55^{*}$ & $2,18^{*}$ & $0,12 *$ & $0,16^{*}$ & $0,71^{*}$ & $1,28 *$ & $0,11^{*}$ & 0,07 & $0,60^{*}$ & $1,14^{*}$ & $0,10^{*}$ & 0,05 & $0,75^{*}$ & $1,55^{*}$ \\
\hline 123 & 0,07 & 0,34 & 0,00 & $5,00 *$ & $0,49 * * *$ & 0,31 & 0,24 & 1,09 & $0,67 *$ & $0,42 *$ & $0,20^{*}$ & $1,44^{*}$ & $0,81^{*}$ & $0,49 *$ & $0,18^{* *}$ & $0,84^{* *}$ & $0,59 *$ & $0,27 *$ & $0,37 *$ & $1,19 *$ \\
\hline 131 & $0,49 * *$ & 0,27 & 0,10 & $5,00 *$ & $0,35^{* *}$ & $0,69 *$ & 0,36 & 0,61 & $0,39 *$ & $0,55^{*}$ & $0,21^{*}$ & $1,18^{*}$ & $0,40^{*}$ & $0,56^{*}$ & 0,14 & $0,84^{*}$ & $0,49 *$ & $0,66^{*}$ & $0,37^{*}$ & $1,10 *$ \\
\hline 132 & 0,06 & $0,41 * *$ & $0,79 *$ & 1,00 & 0,06 & 0,03 & $0,60 *$ & $2,10^{*}$ & $0,14^{*}$ & $0,15^{*}$ & $0,80 *$ & $1,31^{*}$ & $0,12^{*}$ & 0,07 & $0,73^{*}$ & $1,17^{*}$ & $0,10 *$ & 0,04 & $0,76^{*}$ & $1,56^{*}$ \\
\hline 133 & 0,07 & 0,33 & 0,11 & $5,00 *$ & 0,26 & 0,10 & 0,33 & 0,81 & $0,47^{*}$ & $0,26^{*}$ & $0,24^{*}$ & $1,51^{*}$ & $0,41^{*}$ & $0,21^{*}$ & $0,21^{* *}$ & $0,83^{* *}$ & $0,41^{*}$ & $0,15^{*}$ & $0,39 *$ & $1,22 *$ \\
\hline 211 & 0,18 & 0,11 & $0,57^{*}$ & $1,12 * * *$ & 0,18 & $0,45^{* *}$ & $0,59 *$ & 0,34 & $0,17 *$ & $0,26 *$ & $0,55^{*}$ & $0,64 *$ & $0,18^{* *}$ & $0,27^{*}$ & $0,53^{*}$ & $0,45 * *$ & $0,31^{*}$ & $0,42 *$ & $0,65^{*}$ & $0,72 *$ \\
\hline 212 & 0,07 & $0,45^{* *}$ & $0,90 *$ & 1,00 & 0,07 & 0,04 & $0,55^{*}$ & $2,18^{*}$ & $0,12 *$ & $0,16^{*}$ & $0,71^{*}$ & $1,28 *$ & $0,11^{*}$ & 0,07 & $0,60 *$ & $1,14^{*}$ & $0,10^{*}$ & 0,05 & $0,75^{*}$ & $1,55^{*}$ \\
\hline 213 & $1,00^{*}$ & $1,00 * *$ & $0,69 *$ & 1,18 & $0,76^{*}$ & $0,51^{* * *}$ & $0,69 *$ & 0,65 & $0,88^{*}$ & $0,62 *$ & $0,71^{*}$ & $1,01^{*}$ & $0,93 *$ & $0,77 *$ & $0,71^{*}$ & $0,91^{*}$ & $0,73^{*}$ & $0,52^{*}$ & $0,76^{*}$ & $1,07 *$ \\
\hline 221 & $0,45^{* *}$ & 0,22 & 0,00 & $5,00 *$ & $0,31^{* * *}$ & $0,58 *$ & 0,32 & 0,70 & $0,31^{*}$ & $0,42 *$ & $0,19 *$ & $1,26^{*}$ & $0,32 *$ & $0,42 *$ & 0,14 & $0,90 *$ & $0,42 *$ & $0,55^{*}$ & $0,36^{*}$ & $1,08^{*}$ \\
\hline 222 & 0,07 & $0,45^{* *}$ & $0,90 *$ & 1,00 & 0,07 & 0,04 & $0,55^{*}$ & $2,18^{*}$ & $0,12^{*}$ & $0,16^{*}$ & $0,71^{*}$ & $1,28 *$ & $0,11^{*}$ & 0,07 & $0,60 *$ & $1,14^{*}$ & $0,10^{*}$ & 0,05 & $0,75^{*}$ & $1,55^{*}$ \\
\hline 223 & 0,07 & 0,34 & 0,00 & $5,00 *$ & $0,49 * * *$ & 0,31 & 0,24 & 1,09 & $0,67 *$ & $0,42 *$ & $0,20^{*}$ & $1,44^{*}$ & $0,81^{*}$ & $0,49 *$ & $0,18^{* *}$ & $0,84 * *$ & $0,59 *$ & $0,27 *$ & $0,37^{*}$ & $1,19^{*}$ \\
\hline 231 & 0,23 & 0,12 & 0,41 & $1,61^{* *}$ & 0,22 & $0,47^{*}$ & 0,38 & 0,43 & $0,23^{*}$ & $0,31 *$ & $0,26^{*}$ & $0,80^{*}$ & $0,23^{*}$ & $0,31^{*}$ & $0,21 * *$ & $0,58 *$ & $0,36^{*}$ & $0,46^{*}$ & $0,43^{*}$ & $0,80 *$ \\
\hline 232 & 0,08 & $0,46^{* *}$ & $1,00^{*}$ & 1,00 & $0,13^{* * *}$ & 0,05 & $0,90^{*}$ & $1,90 *$ & $0,15^{*}$ & $0,16^{*}$ & $0,90 *$ & $1,18^{*}$ & $0,16^{*}$ & $0,08 * * *$ & $0,91 *$ & $1,07^{*}$ & $0,12 *$ & 0,05 & $0,93^{*}$ & $1,45^{*}$ \\
\hline 233 & $1,00 *$ & $1,00^{* *}$ & 0,37 & 1,02 & $0,75^{*}$ & $0,51^{* * *}$ & $0,46^{* *}$ & 0,56 & $0,86^{*}$ & $0,63^{*}$ & $0,36^{*}$ & $0,96^{*}$ & $0,91^{*}$ & $0,80^{*}$ & $0,31^{*}$ & $0,77^{*}$ & $0,71^{*}$ & $0,57^{*}$ & $0,48^{*}$ & $1,01^{*}$ \\
\hline 311 & $0,45^{* *}$ & 0,22 & 0,02 & $5,00 *$ & $0,31^{* * *}$ & $0,58 *$ & 0,32 & 0,70 & $0,31^{*}$ & $0,42^{*}$ & $0,19 *$ & $1,26^{*}$ & $0,32 *$ & $0,42 *$ & $0,15^{* * *}$ & $0,90 *$ & $0,42^{*}$ & $0,55^{*}$ & $0,36^{*}$ & $1,08^{*}$ \\
\hline 312 & 0,07 & $0,45^{* *}$ & $0,90 *$ & 1,00 & 0,07 & 0,04 & $0,55^{*}$ & $2,18^{*}$ & $0,12^{*}$ & $0,16^{*}$ & $0,71^{*}$ & $1,28 *$ & $0,11^{*}$ & 0,07 & $0,60 *$ & $1,14^{*}$ & $0,10^{*}$ & 0,05 & $0,75^{*}$ & $1,55^{*}$ \\
\hline 313 & 0,07 & 0,34 & 0,02 & $5,00^{*}$ & $0,49 * * *$ & 0,31 & 0,24 & 1,08 & $0,67 *$ & $0,42 *$ & $0,20^{*}$ & $1,44^{*}$ & $0,81^{*}$ & $0,49 *$ & $0,19 * *$ & $0,84 * *$ & $0,59 *$ & $0,27 *$ & $0,38 *$ & $1,19 *$ \\
\hline 321 & $0,45^{* *}$ & 0,22 & 0,00 & $5,00 *$ & $0,31^{* * *}$ & $0,58^{*}$ & 0,32 & 0,70 & $0,31^{*}$ & $0,42^{*}$ & $0,19 *$ & $1,26^{*}$ & $0,32 *$ & $0,42 *$ & 0,14 & $0,90 *$ & $0,42^{*}$ & $0,55^{*}$ & $0,36^{*}$ & $1,08^{*}$ \\
\hline 322 & 0,07 & $0,45^{* *}$ & $0,90 *$ & 1,00 & 0,07 & 0,04 & $0,55^{*}$ & $2,18^{*}$ & $0,12 *$ & $0,16^{*}$ & $0,71^{*}$ & $1,28 *$ & $0,11^{*}$ & 0,07 & $0,60 *$ & $1,14 *$ & $0,10^{*}$ & 0,05 & $0,75^{*}$ & $1,55^{*}$ \\
\hline 323 & 0,07 & 0,34 & 0,00 & $5,00 *$ & $0,49 * * *$ & 0,31 & 0,24 & 1,09 & $0,67 *$ & $0,42 *$ & $0,20 *$ & $1,44^{*}$ & $0,81^{*}$ & $0,49 *$ & $0,18 * *$ & $0,84 * *$ & $0,59 *$ & $0,27 *$ & $0,37 *$ & $1,19 *$ \\
\hline 331 & $0,45^{* *}$ & 0,22 & 0,00 & $5,00 *$ & $0,31^{* * *}$ & $0,57 *$ & 0,30 & 0,69 & $0,31^{*}$ & $0,42 *$ & $0,17 *$ & $1,26^{*}$ & $0,31 *$ & $0,42 *$ & 0,12 & $0,88 *$ & $0,42 *$ & $0,55^{*}$ & $0,36 *$ & $1,07^{*}$ \\
\hline 332 & 0,07 & $0,45 * *$ & $0,89 *$ & 1,00 & 0,07 & 0,04 & $0,54 *$ & $2,14^{*}$ & $0,11^{*}$ & $0,16^{*}$ & $0,67 *$ & $1,28 *$ & $0,10 *$ & 0,07 & $0,53 *$ & $1,14 *$ & $0,09 *$ & 0,05 & $0,69 *$ & $1,56^{*}$ \\
\hline 333 & 0,07 & 0,34 & 0,00 & $5,00 *$ & $0,49 * * *$ & 0,31 & 0,22 & 1,07 & $0,67 *$ & $0,42 *$ & $0,18^{*}$ & $1,45^{*}$ & $0,82 *$ & $0,49 *$ & $0,16^{* * *}$ & $0,85 * *$ & $0,59 *$ & $0,27 *$ & $0,35^{*}$ & $1,20^{*}$ \\
\hline
\end{tabular}

* 1\%; ** 5\%; *** $10 \%$; variáveis em negrito não são significantes. 
Tabela 8 - Análise levando em conta a profissão

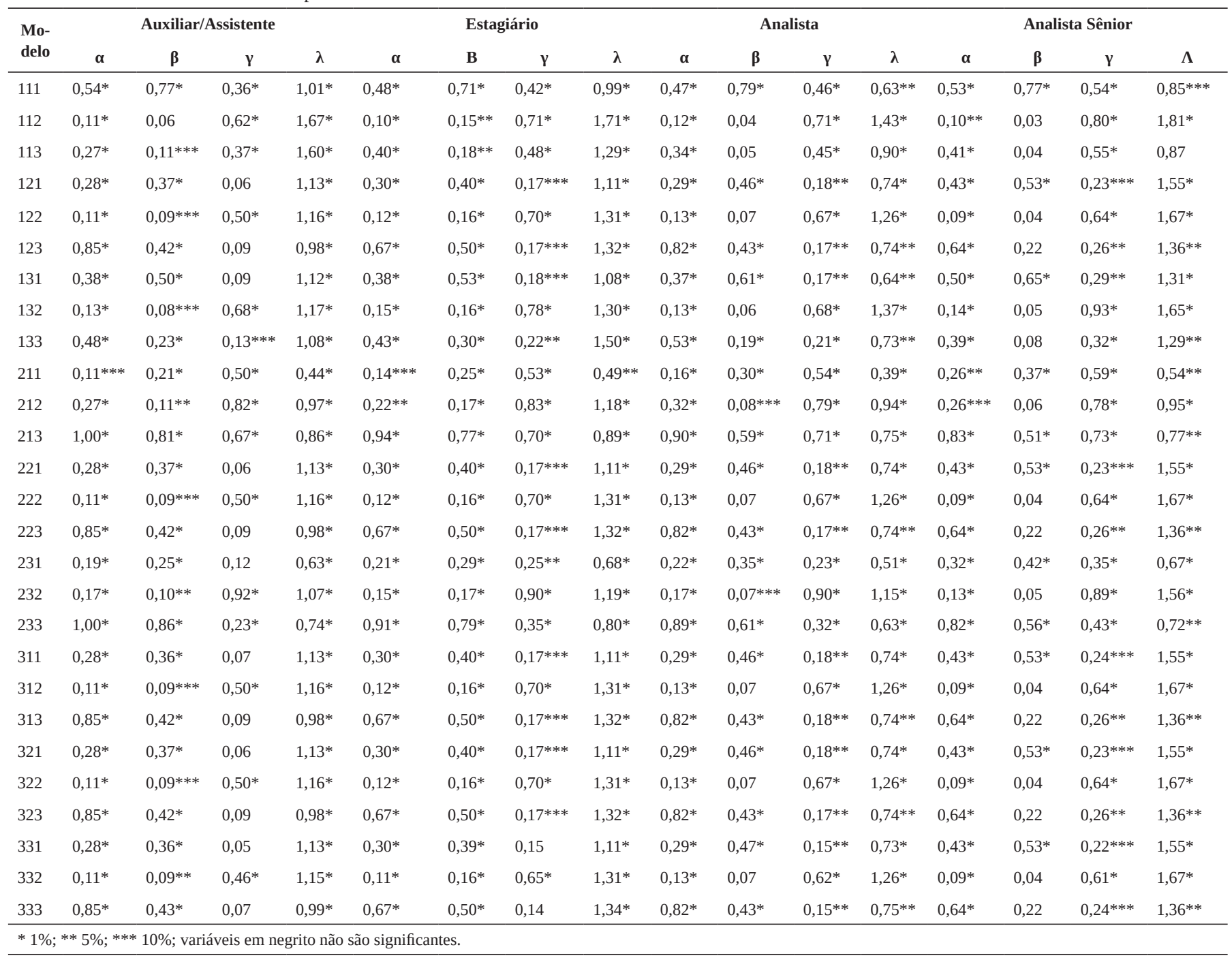


Robert Eugene Lobel et al.

\begin{tabular}{|c|c|c|c|c|c|c|c|c|}
\hline \multirow{2}{*}{$\begin{array}{c}\text { Mode- } \\
\text { los }\end{array}$} & \multicolumn{4}{|c|}{ Supervisão/Coordenação } & \multicolumn{4}{|c|}{ Direção/Gerência } \\
\hline & $\alpha$ & $\beta$ & $Y$ & $\lambda$ & $\alpha$ & $\beta$ & $\mathrm{Y}$ & $\lambda$ \\
\hline 111 & $0,49 *$ & $0,68 *$ & $0,60^{*}$ & $1,58 *$ & $0,62 *$ & $0,80^{*}$ & $0,68 *$ & $1,24^{*}$ \\
\hline 112 & $0,09 *$ & $0,16^{* *}$ & $0,84^{*}$ & $1,70^{*}$ & $0,05 * * *$ & $0,09 * * *$ & $0,82 *$ & $1,58 *$ \\
\hline 113 & $0,29 *$ & $0,22 * *$ & $0,62^{*}$ & $1,64 *$ & $0,16^{* *}$ & $0,15^{* *}$ & $0,69 *$ & $1,97 *$ \\
\hline 121 & $0,39 *$ & $0,48 *$ & $0,37 *$ & $1,64^{*}$ & $0,52 *$ & $0,62 *$ & $0,54 *$ & $1,33^{*}$ \\
\hline 122 & $0,12 *$ & $0,16^{* *}$ & $0,91^{*}$ & $1,66^{*}$ & $0,06^{*}$ & $0,09 * * *$ & $0,88 *$ & $1,57 *$ \\
\hline 123 & $0,46^{*}$ & $0,32 *$ & $0,43^{*}$ & $1,74^{*}$ & $0,32 *$ & $0,24 *$ & $0,55^{*}$ & $1,94^{*}$ \\
\hline 131 & $0,44^{*}$ & $0,58 *$ & $0,42^{*}$ & $1,65^{*}$ & $0,58 *$ & $0,70^{*}$ & $0,54^{*}$ & $1,41^{*}$ \\
\hline 132 & $0,10^{*}$ & $0,16^{* *}$ & $0,82^{*}$ & $1,70^{*}$ & $0,06^{* *}$ & $0,09 * * *$ & $0,86^{*}$ & $1,49 *$ \\
\hline 133 & $0,39 *$ & $0,23 * *$ & $0,42^{*}$ & $1,72 *$ & $0,26^{*}$ & $0,16^{* *}$ & $0,57^{*}$ & $1,92 *$ \\
\hline 211 & $0,29 *$ & $0,37 *$ & $0,66^{*}$ & $0,98 *$ & $0,44^{*}$ & $0,51 *$ & $0,74^{*}$ & $1,12 *$ \\
\hline 212 & $0,27 * *$ & $0,17^{* *}$ & $0,81^{*}$ & $0,97 *$ & $0,16^{* * *}$ & $0,10 * * *$ & $0,87^{*}$ & $1,51^{*}$ \\
\hline 213 & $0,62 *$ & $0,55^{*}$ & $0,77^{*}$ & $1,25^{*}$ & $0,59 *$ & $0,38 *$ & $0,80 *$ & $1,54 *$ \\
\hline 221 & $0,39 *$ & $0,48 *$ & $0,37^{*}$ & $1,64 *$ & $0,52 *$ & $0,62 *$ & $0,54^{*}$ & $1,33^{*}$ \\
\hline 222 & $0,12 *$ & $0,16^{* *}$ & $0,91^{*}$ & $1,66^{*}$ & $0,06^{*}$ & $0,09 * * *$ & $0,88 *$ & $1,57^{*}$ \\
\hline 223 & $0,46^{*}$ & $0,32 *$ & $0,43^{*}$ & $1,74 *$ & $0,32 *$ & $0,24 *$ & $0,55^{*}$ & $1,94 *$ \\
\hline 231 & $0,32 *$ & $0,40 *$ & $0,46^{*}$ & $1,07 *$ & $0,46^{*}$ & $0,54 *$ & $0,63^{*}$ & $1,15^{*}$ \\
\hline 232 & $0,12 *$ & $0,17 * *$ & $0,92^{*}$ & $1,61 *$ & $0,06^{*}$ & $0,10 * * *$ & $0,93 *$ & $1,44^{*}$ \\
\hline 233 & $0,59 *$ & $0,59 *$ & $0,54^{*}$ & $1,24 *$ & $0,54^{*}$ & $0,39 *$ & $0,63^{*}$ & $1,54 *$ \\
\hline 311 & $0,39 *$ & $0,48 *$ & $0,37 *$ & $1,64^{*}$ & $0,52 *$ & $0,62 *$ & $0,54^{*}$ & $1,33^{*}$ \\
\hline 312 & $0,12 *$ & $0,16^{* *}$ & $0,91^{*}$ & $1,66^{*}$ & $0,06^{*}$ & $0,09 * * *$ & $0,88^{*}$ & $1,57 *$ \\
\hline 313 & $0,46^{*}$ & $0,33 *$ & $0,44^{*}$ & $1,75^{*}$ & $0,32 *$ & $0,24 *$ & $0,55^{*}$ & $1,94 *$ \\
\hline 321 & $0,39 *$ & $0,48 *$ & $0,37 *$ & $1,64 *$ & $0,52^{*}$ & $0,62 *$ & $0,54^{*}$ & $1,33^{*}$ \\
\hline 322 & $0,12 *$ & $0,16^{* *}$ & $0,91^{*}$ & $1,66^{*}$ & $0,06^{*}$ & $0,09 * * *$ & $0,88 *$ & $1,57^{*}$ \\
\hline 323 & $0,46^{*}$ & $0,32 *$ & $0,43^{*}$ & $1,74^{*}$ & $0,32 *$ & $0,24 *$ & $0,55^{*}$ & $1,94 *$ \\
\hline 331 & $0,38 *$ & $0,48 *$ & $0,39 *$ & $1,62 *$ & $0,52^{*}$ & $0,62 *$ & $0,53 *$ & $1,31^{*}$ \\
\hline 332 & $0,09 *$ & $0,16^{* *}$ & $0,77^{*}$ & $1,67^{*}$ & $0,06^{*}$ & $0,09 * * *$ & $0,87^{*}$ & $1,57^{*}$ \\
\hline 333 & $0,45^{*}$ & $0,32 *$ & $0,39 *$ & $1,76^{*}$ & $0,32 *$ & $0,25^{*}$ & $0,53^{*}$ & $1,94 *$ \\
\hline
\end{tabular}

*1\%;** 5\%; *** $10 \%$; variáveis em negrito não são significantes.

Fonte: os autores. 


\section{CONCLUSÃO}

O objetivo com este estudo foi analisar as preferências ao risco no ambiente brasileiro seguindo os preceitos da Teoria do Prospecto. Para tal foram estimadas para uma amostra selecionada os parâmetros da função valor e probabilidade supondo diversas formas funcionais, para então associar esses parâmetros a determinadas variáveis sociodemográficas.

No geral, para o público analisado, foi observado que quanto mais se distanciam do ponto de referência, os indivíduos são mais sensíveis às mudanças. Os resultados demonstram a típica forma da função valor em forma de S, ou seja, côncava na região de ganhos e convexa na região de perdas. Entretanto, isso difere para cada modelo.

Ao avaliar as variáveis sociodemográficas, segundo os modelos, nota-se que: em relação ao gênero, na área de ganhos as mulheres são mais avessas ao risco do que os homens; segundo o estado civil, pessoas casadas são mais avessas ao risco tanto na área de ganhos quanto na área de perdas do que pessoas solteiras e divorciadas, além de exibirem uma maior aversão à perda; na análise da faixa etária, mostrou-se uma tendência de pessoas mais velhas serem mais conservadoras; em relação ao nível educacional, pode-se deduzir que um maior nível educacional está associado a uma maior aversão ao risco na área de ganhos e em parte na área de perdas (que não vai de encontro à literatura); na análise pela renda (classe social), notou-se que em geral pessoas com o menor nível de riqueza são mais avessas ao risco; e a análise por profissão não demonstrou resultado.

Ao se comparar os estudos empíricos da literatura, notou-se que a análise por gênero, estado civil, faixa etária e renda mostra relativas similaridades com outros estudos em países desenvolvidos e em desenvolvimento. Entretanto, o nível educacional mostra resultados contrários ao esperado (o que pode ser fundamentado como um viés da amostra) e a análise por profissão chega a resultados inconclusivos.

Para estudos futuros, sugere-se uma aplicação ampla (de caráter nacional), com o intuito de investigar se a questão regional brasileira pode impactar mais ou menos em alguns fatores de percepção ao risco (até porque o Brasil tem proporções continentais e uma grande diversidade cultural e étnica). Neste estudo teve-se como intuito trazer a metodologia com os parâmetros das funções valor (com inclusão da função logarítmica modificada) e peso e investigar primariamente uma amostra inicial no ambiente brasileiro - mas sabe-se que não se pode ter conclusões generalizadas para todo o Brasil (cabe apontar essa limitação). 


\section{REFERÊNCIAS}

ABDELLAOUI, M.; BLEICHRODT, H.; KAMMOUN, H. Do financial professionals behave according to prospect theory? An experimental study. Theory and Decision, v. 74, i. 3, p. 411-429, Sept./Oct. 2011.

ABDELLAOUI, M.; BLEICHRODT, H.; L'HARIDON, O. A tractable method to measure utility and loss aversion under prospect theory. Journal of Risk and Uncertainty, v. 36, i. 3, p. 245-266, June. 2008.

ABDELLAOUI, M.; BLEICHRODT, H.; L'HARIDON, O. Sign-Dependence in Intertemporal Choice. Journal of Risk and Uncertainty, v. 47, i. 3, p. 225-253, Dec. 2013.

ABDELLAOUI, M.; BLEICHRODT, H.; PARASCHIV, C. Loss Aversion Under Prospect Theory: A Parameter-Free Measurement. Management Science, v. 53, i. 10, p. 1659-1674, Oct. 2007.

ALLAIS, M. Le Comportement de l'HommeRationneldevant le Risque: Critique des Postulats et Axiomes de l'EcoleAmericaine. Econometrica, v. 21, n. 4, p. 503-546, Out. 1953.

BARBERIS, N. C.; THALER, R. A survey of behavioral finance. In: CONSTANTINIDES, G. M.; HARRIS, M. et al. (Ed.). Handbook of the Economics of Finance, Elsevier, v. 1, p. 1053-1128, 2003.

BARBERIS, N. C. Thirty Years of Prospect Theory in Economics: A Review and Assessment. Journal of Economic Perspectives, v. 27, i. 1, p. 173-96, 2013.

BARSKY, R. B. et al. Preference Parameters and Behavioral Heterogeneity: An Experimental Approach in the Health and Retirement Study. The Quarterly Journal of Economics, v. 112, i. 2, p. 537-579, May 1997.

BOOIJ, A.; VAN PRAAG, B.; VAN DE KUILEN, G. A parametric analysis of prospect theory's functionals for the general population. Theory and Decision, v. 68, i. 1, p. 115-148, Feb. 2010.

BORGHANS, L. et al. Gender Differences In Risk Aversion And Ambiguity Aversion. Journal of the European Economic Association, v. 7, i. 2-3, p. 649-658, Feb. 2009. 
BRAGA, R. Ganhos e perdas em investimentos: um estudo experimental sobre personalidades à luz da teoria do prospecto e do efeito disposição. 2015. 237 p. Tese (Doutorado em Ciências)-Universidade de São Paulo, São Paulo, 2015.

BUI, T. Prospect Theory and Functional Choice. 2009. Dissertation (Graduate School in Partial Fulfillment of the Requirements for the Degree Erasmus Mundus Master: Models and Methods of Quantitative Economics (QEM))-Bielefeld University and The University of Paris 1 Panthéon, Sorbonne, 2009.

CAMERER, C. An experimental test of several generalized utility theories. Journal of Risk and Uncertainty, v. 2, i. 1, p. 61-104, Apr. 1989.

CAMERER, C. Prospect theory in the wild: Evidence from the field. In: KAHNEMAN, D.; TVERSKY, A. (Ed.). Choices, Values, and Frames. Cambridge, United Kingdom: Cambridge University Press, 2000.

CHARUPAT, N. et al. Emotional balance and probability weighting. Theory and Decision, v. 75, i. 1, p. 17-41, July 2013.

COHEN, A.; EINAV, L. Estimating Risk Preferences from Deductible Choice. American Economic Review, v. 97, i. 3, p. 745-788, June 2007.

CROSON, R.; GNEEZY, U. Gender Differences in Preferences. Journal of Economic Literature, v. 47, i. 2, p. 448-74, June 2009. Disponível em: <http://www. aeaweb.org/articles.php?doi=10.1257/jel.47.2.448>. Acesso em: 10 ago. 2017.

DE BONDT, W. F. M. A portrait of the individual investor. European Economic Review, v. 42, i. 3-5, p. 831-844, May 1998.

DICHTL, H.; DROBETZ, W. Dollar-Cost Averaging and Prospect Theory Investors: An Explanation for a Popular Investment Strategy. Journal of Behavioral Finance, v. 12, i. 1, p. 41-52, Mar. 2011.

DOHMEN, T. et al. Individual risk attitudes: new evidence from a large, representative, experimentally-validated survey. CEPR Discussion Papers, n. 5517. Avalaible from: <http://ssrn.com/abstract=906866>. Acesso em: 10 ago. 2017.

DONKERS, B.; MELENBERG, B.; VAN SOEST, A. Estimating Risk Attitudes using Lotteries: A Large Sample Approach. Journal of Risk and Uncertainty, v. 22, i. 2, p. 165-195, Mar. 2001. 
DONKERS, B.; VAN SOEST, A. Subjective Measures of Household Preferences and Financial Decisions. Journal of Economic Psychology, v. 20, i. 6, p. 613-642, Dec. 1999.

ELLSBERG, D. Risk, Ambiguity, and the Savage Axioms. The Quarterly Journal of Economics, v. 75, i. 4, p. 643-669, Nov. 1961.

ETCHART-VINCENT, N.; L'HARIDON, O. Monetary incentives in the loss domain and behavior toward risk: An experimental comparison of three reward schemes including real losses. Journal of Risk and Uncertainty, v. 42, i. 1, p. 61-83, Feb. 2011.

FEHR-DUDA, H.; GENNARO, M.; SCHUBERT, R. Gender, Financial Risk, and Probability Weights. Theory and Decision, v. 60, i. 2-3, p. 283-313, May 2006.

GÄCHTER, S.; JOHNSON, E. J.; HERRMANN, A. Individual-level loss aversion in riskless and risky choices. CeDEx discussion paper series, i. 2010-2020, 2010.

GOLDSTEIN, W. M.; EINHORN, H. J. Expression theory and the preference reversal phenomena. Psychological Review, US, v. 94, i. 2, p. 236-254, 1987.

GRABLE, J. Financial Risk Tolerance and Additional Factors That Affect Risk Taking in Everyday Money Matters. Journal of Business and Psychology, v. 14, i. 4, p. 625-630, June 2000.

GUREVICH, G.; KLIGER, D.; LEVY, O. Decision-making under uncertainty - A field study of cumulative prospect theory. Journal of Banking \& Finance, v. 33, i. 7, p. 1221-1229, July 2009.

HALEK, M.; EISENHAUER, J. G. Demography of Risk Aversion. The Journal of Risk and Insurance, v. 68, i. 1, p. 1-24, Mar. 2001.

HARRISON, G. W.; HUMPHREY, S. J.; VERSCHOOR, A. Choice under Uncertainty: Evidence from Ethiopia, India and Uganda. The Economic Journal, v. 120, i. 543, p. 80-104, Mar. 2010.

HARRISON, G. W.; LAU, M. I.; RUTSTRÖM, E. E. Estimating Risk Attitudes in Denmark: A Field Experiment. Scandinavian Journal of Economics, v. 109, i. 2, p. 341-368, June 2007. 
HARRISON, G. W.; RUTSTRÖM, E. Expected utility theory and prospect theory: one wedding and a decent funeral. Experimental Economics, v. 12, i. 2, p. 133-158, June 2009.

HARTOG, J.; FERRER-I-CARBONELL, A.; JONKER, N. Linking Measured Risk Aversion to Individual Characteristics. Kyklos, v. 55, i. 1, p. 3-26, Nov. 2002.

IBGE. Pesquisa Nacional por Amostra de Domicílios (Pnad) Contínua. Brasília, DF: IBGE, 2014.

IRWIN, C. Adolescence and risk taking: How are they related? In: BELL, N. J.; BELL, R. W. (Ed.). Adolescent risk taking. Newbury Park, CA: Sage Publications, 1993.

KAHNEMAN, D.; TVERSKY, A. Prospect Theory: An Analysis of Decision under Risk. Econometrica, v. 47, i. 2, p. 263-291, Mar. 1979.

KARMARKAR, U. S. Subjectively weighted utility: A descriptive extension of the expected utility model. Organizational Behavior and Human Performance, v. 21, i. 1, p. 61-72, Feb. 1978.

KARMARKAR, U. S. Subjectively weighted utility and the Allais Paradox. Organizational Behavior and Human Performance, v. 24, i. 1, p. 67-72, Aug. 1979.

KLIGER, D.; LEVY, O. Theories of choice under risk: Insights from financial markets. Journal of Economic Behavior and Organization, v. 71, i. 2, p. 330-346, Aug. 2009.

KÖBBERLING, V.; WAKKER, P. P. An index of loss aversion. Journal of Economic Theory, v. 122, i. 1, p. 119-131, May 2005.

LOVE, D. A. The Effects of Marital Status and Children on Savings and Portfolio Choice. Review of Financial Studies, v. 23, i. 1, p. 385-432, Jan. 2010.

MARKOWITZ, H. Portfolio Selection. The Journal of Finance, v. 7, i. 1, p. 77-91, Mar. 1952.

NOUSSAIR, C. N.; TRAUTMANN, S. T.; VAN DE KUILEN, G. Higher Order Risk Attitudes, Demographics, and Financial Decisions. The Review of Economic Studies, v. 81, i. 1, p. 325-355, Jan. 2014. 
PÅLSSON, A. M. Does the degree of relative risk aversion vary with household characteristics? Journal of Economic Psychology, v. 17, i. 6, p. 771-787, Dec. 1996.

PRELEC, D. The Probability Weighting Function. Econometrica, v. 66, i. 3, p. 497527, May 1998.

RIEGER, M. O.; BUI, T. Too Risk-Averse for Prospect Theory? Modern Economy, v. 2, i. 4, p. 691-670, Dec. 2011.

RIEGER, M. O.; WANG, M.; HENS, T. Prospect Theory Around the World. NHH Dept. of Finance \& Management Science Discussion Paper, n. 2011-2019, Oct. 2011. Disponível em: <http://ssrn.com/abstract=1957606>. Acesso em: 20 out. 2017.

RIEGER, M. O.; WANG, M. Prospect theory for continuous distributions. Journal of Risk and Uncertainty, v. 36, i. 1, p. 83-102, Jan. 2008.

ROUSSANOV, N. L.; SAVOR, P. G. Marriage and Managers' Attitudes to Risk. San Diego Meetings Paper, AFA, 2013.

SNELBECKER, G. E.; ROSZKOWSKI, M. J.; CUTLER, N. E. Investors’ Risk Tolerance and Return Aspirations, and Financial Advisors' Interpretations: A Conceptual Model and Exploratory Data. Journal of Behavioral Economics, v. 19, i. 4, p. 377-393, 1990.

SUNDÉN, A. E.; SURETTE, B. J. Gender Differences in the Allocation of Assets in Retirement Savings Plans. The American Economic Review, v. 88, i. 2, p. 207-211, May 1998.

TANAKA, T.; CAMERER, C. F.; NGUYEN, Q. Risk and Time Preferences: Linking Experimental and Household Survey Data from Vietnam. American Economic Review, v. 100, i. 1, p. 557-71, Mar. 2010.

TVERSKY, A.; KAHNEMAN, D. Advances in prospect theory: Cumulative representation of uncertainty. Journal of Risk and Uncertainty, v. 5, i. 4, p. 297-323, 1992.

VON GAUDECKER, H.-M.; VAN SOEST, A.; WENGSTROM, E. Heterogeneity in Risky Choice Behavior in a Broad Population. American Economic Review, v. 101, i. 2, p. 664-94, Apr. 2011. 
ZEISBERGER, S.; VRECKO, D.; LANGER, T. Measuring the time stability of Prospect Theory preferences. Theory and Decision, v. 72, i. 3, p. 359-386, Mar. 2012.

\section{Como citar este artigo:}

\section{ABNT}

LOBEL, Robert Eugene et al. Teoria do Prospecto: fatores determinantes nas preferências ao risco no Brasil. RACE, Revista de Administração, Contabilidade e Economia, Joaçaba: Ed. Unoesc, v. 17, n. 2, p. 535-566, maio/ago. 2018. Disponível em: <http://editora.unoesc.edu.br/index.php/race>. Acesso em: dia/mês/ano.

APA

Lobel, R. E., Klotzle, M. C., Silva, P. V. J. da G., \& Pinto, A. C. F. (2018). Teoria do Prospecto: fatores determinantes nas preferências ao risco no Brasil. RACE, Revista de Administração, Contabilidade e Economia, 17(2), 535-566. Recuperado em dia/ mês/ano, de http://editora.unoesc.edu.br/index.php/race 
